\title{
On the streaming model for redshift-space distortions
}

\author{
Joseph Kuruvilla ${ }^{1 \star} \dagger$ and Cristiano Porciani ${ }^{1} \ddagger$ \\ ${ }^{1}$ Argelander-Institut für Astronomie, Auf dem Hügel 71, D-53121 Bonn, Germany
}

Accepted XXX. Received YYY; in original form ZZZ

\begin{abstract}
The streaming model describes the mapping between real and redshift space for 2point clustering statistics. Its key element is the probability density function (PDF) of line-of-sight pairwise peculiar velocities. Following a kinetic-theory approach, we derive the fundamental equations of the streaming model for ordered and unordered pairs. In the first case, we recover the classic equation while we demonstrate that modifications are necessary for unordered pairs. We then discuss several statistical properties of the pairwise velocities for DM particles and haloes by using a suite of high-resolution $N$-body simulations. We test the often used Gaussian ansatz for the PDF of pairwise velocities and discuss its limitations. Finally, we introduce a mixture of Gaussians which is known in statistics as the generalised hyperbolic distribution and show that it provides an accurate fit to the PDF. Once inserted in the streaming equation, the fit yields an excellent description of redshift-space correlations at all scales that vastly outperforms the Gaussian and exponential approximations. Using a principal-component analysis, we reduce the complexity of our model for large redshiftspace separations. Our results increase the robustness of studies of anisotropic galaxy clustering and are useful for extending them towards smaller scales in order to test theories of gravity and interacting dark-energy models.
\end{abstract}

Key words: cosmology: large-scale structure of Universe, theory; galaxies: distances and redshifts; methods: analytical, statistical

\section{INTRODUCTION}

Galaxy redshift surveys provide us with three-dimensional maps of the Universe. However, the resulting charts are twisted by the fact that we convert redshifts into distances by assuming a homogeneous model of cosmic expansion. It is well known that the measured redshift is not a perfect distance indicator in the presence of density perturbations. Peculiar galaxy motions along the line of sight (los) generate the leading corrections and give rise to the so-called 'redshift-space distortions' (RSD) between the reconstructed configuration (in 'redshift space') and the actual galaxy distribution (in 'real' space).

The clustering pattern in redshift space predominantly showcases two spurious features. Collapsed structures appear highly elongated along the los due to the velocity dispersion of their constituent galaxies, a phenomenon known as the 'Finger-of-God' (FoG) effect (Jackson 1972; Sargent \& Turner 1977). At the same time, large-scale flows towards

\footnotetext{
* E-mail: joseph@astro.uni-bonn.de

$\dagger$ Member of the International Max Planck Research School (IMPRS) for Astronomy and Astrophysics at the Universities of Bonn and Cologne

‡ E-mail: porciani@astro.uni-bonn.de
}

overdense regions (or away from underdense regions) coherently deform the inferred galaxy distribution (Kaiser 1987). Overall, RSD break the rotational invariance of galaxy $N$ point statistics which are anisotropic functions of the galaxy separations (in redshift space) along the los and in the plane of the sky. On large scales, the degree of anisotropy reflects the growth rate of cosmic structure and can thus be used to probe dark energy and gravity theories (see Hamilton 1998, for a comprehensive review).

RSD are clearly detected in measurements of galaxy clustering (e.g. Davis \& Peebles 1983; Fisher et al. 1994; Peacock et al. 2001; Guzzo et al. 2008) and forthcoming surveys have the potential to extract valuable cosmological information from them (White et al. 2009; Giannantonio et al. 2012; Borzyszkowski et al. 2017). The main limiting factor is modeling galaxy statistics in redshift space to the required accuracy for the widest possible range of scales. This is a formidable problem involving four non-linear quantities: the density and the velocity fields, galaxy biasing and the mapping from real to redshift space. Several lines of research have been pursued over the last decades with the aim of improving our understanding of RSD, including perturbative approaches for the largest scales (e.g Matsubara 2008; Taruya et al. 2009, 2013; Senatore \& Zaldarriaga 2014), phenomeno- 
logical models (e.g. Peacock \& Dodds 1994), and combinations of the two (e.g. Reid \& White 2011).

In this paper, we focus on the phenomenological approach introduced by Peebles (1980) to model the galaxy two-point correlation function in redshift space and subsequently generalised by Fisher (1995, who dubbed it the 'streaming model'). In this framework, the mapping from real to redshift space is discussed in terms of the pairwise velocity distribution function. Basically, (one plus) the anisotropic correlation function in redshift space is written as an integral of (one plus) the spherically-symmetric correlation function in real space times the probability density of the relative los peculiar velocity. This model is exact in the distant-observer limit. Its properties in Fourier space have been thoroughly investigated by Scoccimarro (2004).

The history of the streaming model is rich and varied. In its early applications, it was used to model the smallscale clustering of galaxies from the CfA survey (Davis \& Peebles 1983). The pairwise velocity distribution was assumed to be exponential ${ }^{1}$. The (scale-dependent) mean pairwise (or streaming) velocity along the los was determined by requiring the conservation of galaxy pairs under the stableclustering hypothesis while the corresponding velocity dispersion (assumed to be scale independent to first approximation) was treated as a free parameter and measured from the suppression of the redshift-space correlations along the los at fixed transverse separation (e.g. Davis \& Peebles 1983; Bean et al. 1983; Li et al. 2006). Later on, Fisher (1995, see also Fisher et al. 1994) demonstrated that the streaming model with a Gaussian velocity distribution and a scale-dependent velocity dispersion is compatible with linear (Eulerian) perturbation theory on large scales. Therefore, it became clear that the character of the velocity PDF must change substantially with the galaxy separation, which makes the development of accurate theoretical models difficult. For this reason, hybrid models that combine linear perturbative predictions in redshift space with a (scale-independent) streaming term for the incoherent small-scale motions were introduced in order to describe galaxy clustering on a wider range of separations (Peacock \& West 1992; Park et al. 1994; Peacock \& Dodds 1994). These 'dispersion models' continue to be very popular (e.g. Hawkins et al. 2003; Guzzo et al. 2008; Beutler et al. 2012; Chuang \& Wang 2013) although they correspond to a streaming model with a discontinous velocity PDF (Scoccimarro 2004) and may lead to biased estimates of the cosmological parameters (Kwan et al. 2012).

Over time, the 'Gaussian streaming model' (GSM) has become the workhorse of RSD studies (Reid et al. 2012; Samushia et al. 2014; Alam et al. 2015; Chuang et al. 2017; Satpathy et al. 2017). It requires three inputs: the realspace correlation function plus the mean and the dispersion of the los relative velocity distribution both as a function of the spatial separation and the orientation of the pairs. Several flavours of perturbation theory have been used to model these basic ingredients obtaining satisfactory agreement with $N$-body simulations at least for certain redshifts, tracers and scales (Reid \& White 2011; Reid et al. 2012;

1 Other analytical forms have also been considered but the exponential provided the best fit to the observational data.
Carlson et al. 2013; Wang et al. 2014; White 2014; Vlah et al. 2016; Kopp et al. 2016).

Analytical considerations and numerical simulations show that the los pairwise velocity distribution is strongly non-Gaussian at all scales, being characterized by a net skewness and approximately exponential tails (Efstathiou et al. 1988; Fisher et al. 1994; Juszkiewicz et al. 1998; Scoccimarro 2004). Different strategies have been employed to explain this shape ranging from the halo model (Sheth 1996; Sheth \& Diaferio 2001; Tinker 2007) to the superposition of environment-dependent Gaussian or quasi-Gaussian distributions (Bianchi et al. 2015, 2016). Given the non-Gaussian nature of the velocity PDF, the GSM corresponds to a cumulant expansion truncated at second order (Reid \& White 2011). An approximate extension to the third cumulant has been presented using the Edgeworth expansion around a Gaussian at first order (Uhlemann et al. 2015).

After discussing the limitations of the GSM, we introduce a new parameterization for the pairwise velocity PDF and show that it accurately reproduces the ouptut of $N$ body simulations both at the level of particles and haloes as well as for their 2-point correlation functions in redshift space. There is a growing interest in extending RSD studies to smaller scales as a test of modified gravity and interacting dark energy models (e.g. Jennings et al. 2012; Marulli et al. 2012; Hellwing et al. 2014; Taruya et al. 2014; Zu et al. 2014; Xu 2015; Barreira et al. 2016; Sabiu et al. 2016; Arnalte-Mur et al. 2017). These future developments provide the main motivation for our work.

The paper is organized as follows. In Sections 2 and 3, we introduce the suite of $N$-body simulations used for our study and the basic principles of redshift-space distortions respectively. In Section 4, we present an original derivation of the streaming model starting from the 2-particle distribution function in phase space. We show that the model is regulated by different equations depending on whether one is considering ordered or unordered pairs. Here, we also review the applications of the streaming model to galaxy redshift surveys and test the basic assumptions of the GSM against $N$-body simulations. In Section 5, we illustrate how the relative los velocity and its cumulants are connected to the (isotropic) radial and tangential components of the pairwise velocity vector. We then characterise the scale dependence of the first 4 cumulants for dark-matter (DM) particles and haloes extracted from our numerical simulations. This allows us to discuss the pairwise velocity bias for the haloes. Further, we use the $N$-body simulations to decompose the matter pairwise velocity distribution into the contributions generated by DM haloes of different masses. In Section 6 , we introduce the generalized hyperbolic distribution to model the PDF of the los pairwise velocity and show that it vastly improves upon previous approximations for both DM particles and haloes. Finally, in Section 7, we summarise our main achievements and conclude.

\section{$2 \quad N$-BODY SIMULATIONS}

Our study combines analytical and numerical work. For the latter, we consider six $N$-body simulations run with the code GADGET-2 (Springel et al. 2001; Springel 2005). As a reference, we use the zero-redshift output of the simulation 
Table 1. Cosmological parameters characterizing our $N$-body simulations.

\begin{tabular}{cccccccc}
\hline Name & $f_{\mathrm{NL}}$ & $h$ & $\sigma_{8}$ & $n_{\mathrm{s}}$ & $\Omega_{\mathrm{m}}$ & $\Omega_{\mathrm{b}}$ & $\Omega_{\Lambda}$ \\
\hline W0 & 0 & 0.7010 & 0.8170 & 0.9600 & 0.2790 & 0.0462 & 0.7210 \\
Wf $f_{\mathrm{NL}}$ & $\pm 27, \pm 80$ & 0.7010 & 0.8170 & 0.9600 & 0.2790 & 0.0462 & 0.7210 \\
P0 & 0 & 0.6774 & 0.8159 & 0.9667 & 0.3089 & 0.0486 & 0.6911 \\
\hline
\end{tabular}

that was labelled 1.0 in Pillepich et al. (2010) and we now dub W0. This run follows the formation of the large-scale structure from Gaussian initial conditions within a periodic cubic box with a side of $1200 h^{-1} \mathrm{Mpc}$. It assumes a flat $\Lambda \mathrm{CDM}$ cosmology with the best-fitting parameters determined by the 5-yr analysis of the WMAP mission (Komatsu et al. 2009) and considers $1024^{3}$ particles with a mass of $1.246 \times 10^{11} h^{-1} \mathrm{M}_{\odot}$ (see Pillepich et al. 2010 for further details). To study the redshift evolution of our results, we consider other snapshots extracted from the same run. Furthermore, in order to explore the sensitivity of our findings to the underlying cosmological model and to the properties of the linear density perturbations, we use other five simulations. Four of them $(\mathrm{W}-80, \mathrm{~W}-27, \mathrm{~W}+27, \mathrm{~W}+80)$ are also presented in Pillepich et al. (2010). Their only difference with respect to our reference run is the presence of non-Gaussian initial conditions of the local type with $f_{\mathrm{NL}}=-80,-27,+27$ and +80 . The remaining simulation $(\mathrm{P} 0)$, instead, has been run specifically for this work and assumes the best-fitting cosmological parameters from the Planck mission (Planck Collaboration et al. 2016). In all cases, we use the same box and softening lengths as well as the same number of particles and initial redshift. This implies that the $\mathrm{P} 0$ run has a slightly higher particle mass, $1.379 \times 10^{11} h^{-1} \mathrm{M}_{\odot}$. A comparison of the cosmological parameters used in the simulations is given in Table. 1 .

We identify DM haloes using the ROCKSTAR halo finder (Behroozi et al. 2013) in the default configuration and only consider bound objects containing at least 100 particles within the virial radius.

\section{REDSHIFT-SPACE DISTORTIONS}

We generally use redshift as a distance indicator assuming a homogeneous model of cosmic expansion with instantaneous scale factor $a$ and Hubble parameter $H$. However, in the presence of peculiar velocities, we need to distinguish between the redshift-inferred distance of a generic tracer of the large-scale structure of the Universe (e.g. a galaxy or a galaxy cluster) $\boldsymbol{x}_{\mathrm{s}}$ and its true comoving distance $\boldsymbol{x}$. In the distant-observer (or plane-parallel) approximation (Hamilton 1998),

$\boldsymbol{x}_{\mathrm{S}}=\boldsymbol{x}+(\boldsymbol{v} \cdot \hat{z}) \hat{z}$

where $\boldsymbol{v}$ denotes the peculiar velocity divided by the factor $a H$ and $\hat{z}$ denotes the los direction. The spurious displacement along the los distorts the clustering pattern of the tracers in redshift space from its actual configuration in real space. Let us consider two tracers with real-space separation $\boldsymbol{r}=\boldsymbol{x}_{2}-\boldsymbol{x}_{1}$. The los component of their separation in redshift space is then

$s_{\|}=\left(\boldsymbol{x}_{\mathrm{s}_{2}}-\boldsymbol{x}_{\mathrm{s}_{1}}\right) \cdot \hat{z}=r_{\|}+w_{\|}$, where $r_{\|}=\boldsymbol{r} \cdot \hat{z}$ and $w_{\|}=\left(\boldsymbol{v}_{2}-\boldsymbol{v}_{1}\right) \cdot \hat{z}$, while the transverse separation remains unchanged, i.e.

$s_{\perp}=r_{\perp}$.

If the spatial distribution of the tracers is statistically homogeneous in real space and their two-point correlation function $\xi(r)$ is invariant under rotations of the separation vector $\boldsymbol{r}$, it follows that the correlation function in redshift space $\xi_{\mathrm{S}}\left(s_{\perp}, s_{\|}\right)$is anisotropic between the parallel and transverse components and does not depend on $s=\left(s_{\|}^{2}+s_{\perp}^{2}\right)^{1 / 2}$. An illustrative example is shown in Fig. 1 where we compare $\xi$ and $\xi_{\mathrm{s}}$ for the DM particles in our $N$-body simulation (we have used one of the box axes as the los direction). Note that the iso-correlation contours for $\xi_{\mathrm{s}}$ are elongated along $s_{\|}$at small transverse separations as a manifestation of the FoG effect and are squashed towards the bottom at large $s_{\perp}$ because of coherent infall motions as described in Kaiser (1987).

\section{THE STREAMING MODEL}

The streaming model has been introduced to map $\xi(r)$ on to $\xi_{\mathrm{s}}(\boldsymbol{s})$ (Peebles 1980; Fisher 1995; Scoccimarro 2004). Its basic equation is generally written as

$1+\xi_{\mathrm{s}}\left(s_{\perp}, s_{\|}\right)=\int_{-\infty}^{+\infty}[1+\xi(r)] \mathcal{P}_{w_{\|}}\left(s_{\|}-r_{\|} \mid \boldsymbol{r}\right) \mathrm{d} r_{\|}$,

where $r^{2}=s_{\perp}^{2}+r_{\|}^{2}$ and $\mathcal{P}_{w_{\|}}$denotes the distribution function of the pairwise los velocity at fixed real-space separation vector $\boldsymbol{r}=\left(s_{\perp}, r_{\|}\right)$(i.e. a PDF which is differential in $\left.w_{\|}\right)$. In the remainder of this section, we will show that this classic result is exact provided that ordered pairs are used to define the correlation function and the correct definition of the velocity $\mathrm{PDF}$ is employed.

\subsection{Ordered and unordered pairs}

Two-point correlation functions are statistics of tracer pairs. A pair is a set composed of two elements. Still, we can define two kinds of pairs. If the order in which the elements appear in the pair is important (i.e. it makes sense to define a first element and a second element), we speak of an ordered pair (or 2-tuple). On the other hand, if the order does not matter, we speak of an unordered pair (or pair set). Ordered pairs can be represented by directed graphs (and vice versa), e.g. $A \rightarrow B \neq B \rightarrow A$, and unordered pairs by undirected graphs, e.g. $A-B=B-A$. From a set of $N$ discrete objects, we can form $N(N-1)$ ordered pairs and $N(N-1) / 2$ unordered pairs.

If the two-point correlation function is built out of ordered pairs, the spatial separations $r_{\|}$and $s_{\|}$must be signed numbers (while $s_{\perp}=r_{\perp} \geq 0$ as they give the magnitude of 


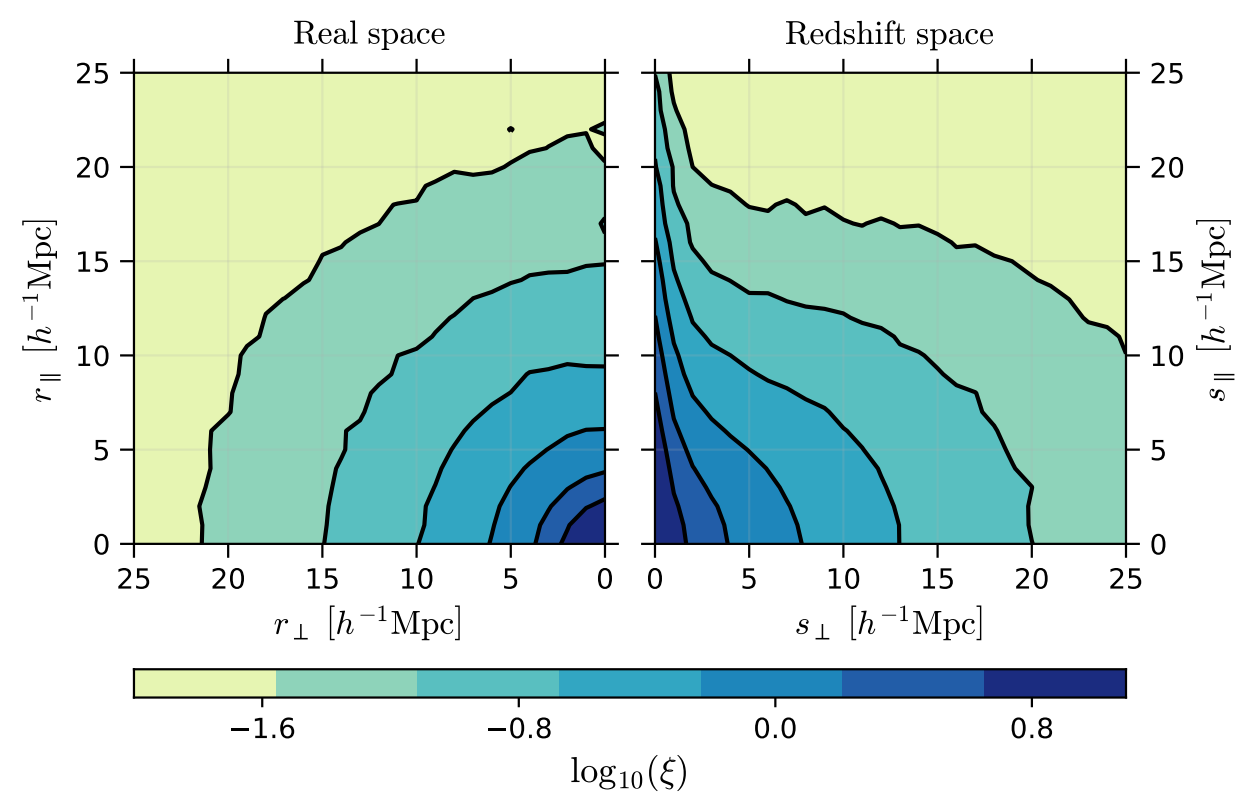

Figure 1. Two-point correlation function in real (left) and redshift space (right) for the DM particles in the W0 simulation at $z=0$. The contour levels correspond to values of $\xi$ and $\xi_{\text {s }}$ ranging from 0.08 (outermost) to 2.56 (innermost) and differing by a factor of 2 between two consecutive levels. To facilitate comparisons, the same levels are used in all figures showing correlation functions.

the two-dimensional vector $\left.\boldsymbol{s}_{\perp}=\boldsymbol{r}_{\perp}\right)$. On the other hand, for unordered pairs, $r_{\|}$and $s_{\|}$are unsigned numbers.

\subsection{The streaming model for ordered pairs}

Let us consider a system of $N$ particles with instantaneous comoving positions $\mathbf{x}_{i}$ and rescaled peculiar velocities $\boldsymbol{v}_{i}$ (where the subscript $i$ identifies the different particles). Following a standard procedure in classical statistical mechanics, we introduce the one- and two-particle phase-space densities (Yvon 1935)

$$
\begin{aligned}
f_{1}(\boldsymbol{x}, \boldsymbol{v})=\left\langle\hat{f}_{1}(\boldsymbol{x}, \boldsymbol{v})\right\rangle= & \left\langle\sum_{i=1}^{N} \delta_{\mathrm{D}}^{(3)}\left(\boldsymbol{x}-\boldsymbol{x}_{i}\right) \delta_{\mathrm{D}}^{(3)}\left(\boldsymbol{v}-\boldsymbol{v}_{i}\right)\right\rangle, \\
f_{2}\left(\boldsymbol{x}_{\mathrm{A}}, \boldsymbol{x}_{\mathrm{B}}, \boldsymbol{v}_{\mathrm{A}}, \boldsymbol{v}_{\mathrm{B}}\right)= & \left\langle\sum_{i=1}^{N} \sum_{j \neq i} \delta_{\mathrm{D}}^{(3)}\left(\boldsymbol{x}_{\mathrm{A}}-\boldsymbol{x}_{i}\right) \delta_{\mathrm{D}}^{(3)}\left(\boldsymbol{x}_{\mathrm{B}}-\boldsymbol{x}_{j}\right)\right. \\
& \left.\delta_{\mathrm{D}}^{(3)}\left(\boldsymbol{v}_{\mathrm{A}}-\boldsymbol{v}_{i}\right) \delta_{\mathrm{D}}^{(3)}\left(\boldsymbol{v}_{\mathrm{B}}-\boldsymbol{v}_{j}\right)\right\rangle,
\end{aligned}
$$

where $\hat{f}_{1}(\boldsymbol{x}, \boldsymbol{v})$ is the discrete one-particle phase density (also known as the Klimontovich or the microscopic density), the brackets denote averaging over an ensemble of realisations and $\delta_{\mathrm{D}}^{(n)}(\mathbf{x})$ is the Dirac delta distribution in $\mathbb{R}^{n}$. Note that $f_{1}$ is normalised to the total number of particles, i.e. $\int f_{1} \mathrm{~d}^{3} x \mathrm{~d}^{3} v=N$ and $f_{2}$ to the total number of ordered pairs, i.e. $\int f_{2} \mathrm{~d}^{3} x_{\mathrm{A}} \mathrm{d}^{3} x_{\mathrm{B}} \mathrm{d}^{3} v_{\mathrm{A}} \mathrm{d}^{3} v_{\mathrm{B}}=N(N-1)$. By definition, the spatial two-point correlation function of the particles is

$1+\xi\left(\mathbf{x}_{\mathrm{A}}, \mathbf{x}_{\mathrm{B}}\right)=\frac{\int f_{2} \mathrm{~d}^{3} v_{\mathrm{A}} \mathrm{d}^{3} v_{\mathrm{B}}}{\left(\int f_{1} \mathrm{~d}^{3} v\right)^{2}}$.

Assuming statistical isotropy (i.e. the invariance under rotations of the expectations over the ensemble) implies that $f_{1}$ can only depend on $x^{2}, v^{2}$ and $\mathbf{x} \cdot \boldsymbol{v}$. Requiring that ensemble averages are also invariant under translations (statistical homogeneity) fixes the dependence of the one-particle distribution function to $f_{1}=\bar{n} F\left(v^{2}\right)$ where the constant $\bar{n}=\int f_{1} \mathrm{~d}^{3} v$ gives the mean particle number density per unit volume and $F$ is an arbitrary function such that $4 \pi \int F\left(v^{2}\right) v^{2} \mathrm{~d} v=1$. Under the same assumptions and for $N \gg 1, \int f_{2} \mathrm{~d}^{3} v_{\mathrm{A}} \mathrm{d}^{3} v_{\mathrm{B}}=$ $\bar{n}^{2}[1+\xi(r)]$.

Our goal is to introduce a new set of distribution functions which are defined in 'redshift phase space'. This can be easily achieved by performing the change of coordinates given in equations (1), (2) and (3):

$$
\begin{aligned}
g_{1}\left(\mathbf{x}_{\mathrm{s}}, \boldsymbol{v}\right) & =f_{1}\left(\mathbf{x}_{\perp}, x_{\mathrm{s} \|}-v_{\|}, \boldsymbol{v}\right)=\bar{n} F\left(v^{2}\right), \\
g_{2}\left(\boldsymbol{s}_{\perp}, s_{\|}, \boldsymbol{v}_{\mathrm{A}}, \boldsymbol{v}_{\mathrm{B}}\right) & =f_{2}\left(\boldsymbol{s}_{\perp}, s_{\|}-v_{\mathrm{B} \|}+v_{\mathrm{A} \|}, \boldsymbol{v}_{\mathrm{A}}, \boldsymbol{v}_{\mathrm{B}}\right) .
\end{aligned}
$$

The spatial two-point correlation function in redshift-space is then

$$
1+\xi_{\mathrm{s}}\left(\boldsymbol{s}_{\perp}, s_{\|}\right)=\frac{\int g_{2} \mathrm{~d}^{3} v_{\mathrm{A}} \mathrm{d}^{3} v_{\mathrm{B}}}{\left(\int g_{1} \mathrm{~d}^{3} v\right)^{2}}=\frac{\int g_{2} \mathrm{~d}^{3} v_{\mathrm{A}} \mathrm{d}^{3} v_{\mathrm{B}}}{\bar{n}^{2}} .
$$

We now rewrite the rhs of equation (9) as

$\int_{-\infty}^{+\infty} f_{2}\left(s_{\perp}, s_{\|}-w_{\|}, \boldsymbol{v}_{\mathrm{A}}, \boldsymbol{v}_{\mathrm{B}}\right) \delta_{\mathrm{D}}^{(1)}\left(w_{\|}-v_{\mathrm{B} \|}+v_{\mathrm{A} \|}\right) \mathrm{d} w_{\|}$,

and we substitute it in equation (10). After multiplying the rhs of the resulting equation by the ratio

$$
\frac{\left.\bar{n}^{2}\left[1+\xi\left(\sqrt{s_{\perp}^{2}+\left(s_{\|}-w_{\|}\right.}\right)^{2}\right)\right]}{\int f_{2}\left(\mathbf{s}_{\perp}, s_{\|}-w_{\|}, \boldsymbol{v}_{\mathrm{A}}, \boldsymbol{v}_{\mathrm{B}}\right) \mathrm{d}^{3} v_{\mathrm{A}} \mathrm{d}^{3} v_{\mathrm{B}}},
$$

(which is identically equal to one), we re-arrange the terms to define the relative line-of-sight velocity PDF for ordered 

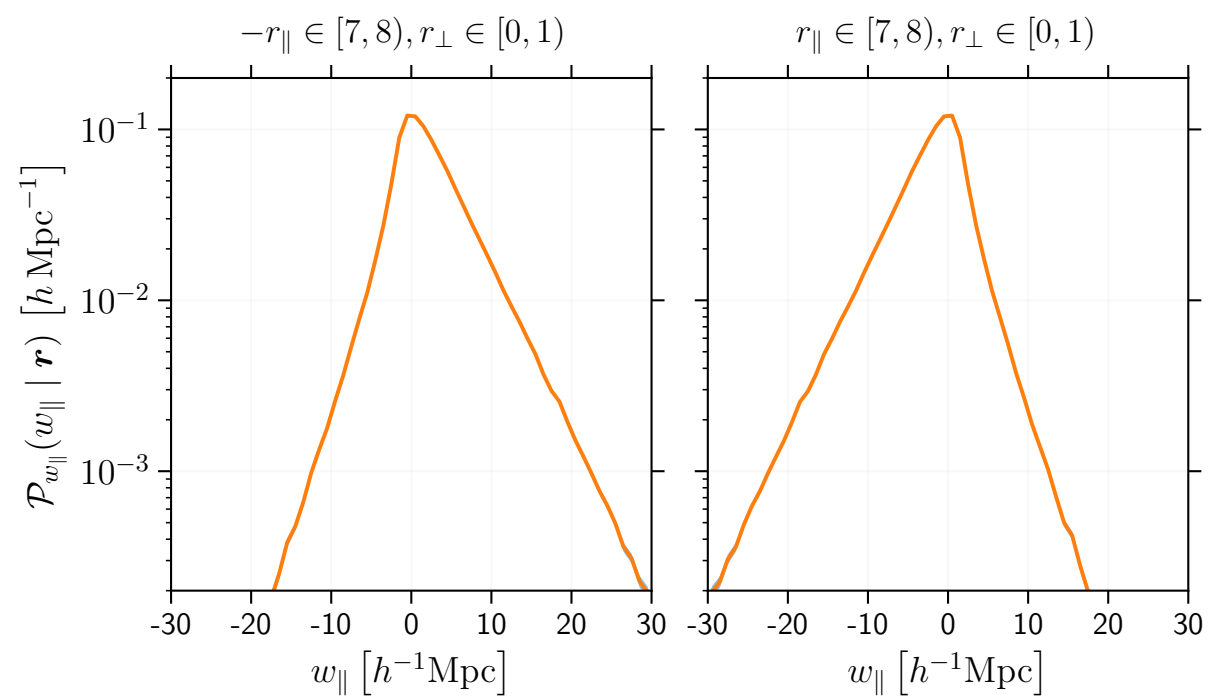

Figure 2. PDF of the relative los velocity for ordered pairs with fixed real-space separation (as indicated by the top labels in units of $\left.h^{-1} \mathrm{Mpc}\right)$. The left- and right-hand side panels correspond to a particle exchange and show the symmetry $\mathcal{P}_{\boldsymbol{w}_{\|}}\left(\boldsymbol{w}_{\|} \mid r_{\perp}, r_{\|}\right)=\mathcal{P}_{\boldsymbol{w}_{\|}}\left(-\boldsymbol{w}_{\|} \mid\right.$ $\left.r_{\perp},-r_{\|}\right)$.

pairs with real-space separation $\boldsymbol{r}=\left(\boldsymbol{s}_{\perp}, s_{\|}-w_{\|}\right)$as

$$
\begin{gathered}
\mathcal{P}_{w_{\|}}\left(w_{\|} \mid \boldsymbol{r}\right)=\frac{\int f_{2}\left(\boldsymbol{r}, \boldsymbol{v}_{\mathrm{A}}, \boldsymbol{v}_{\mathrm{B}}\right) \delta_{\mathrm{D}}^{(1)}\left(w_{\|}-v_{\mathrm{B} \|}+v_{\mathrm{A} \|}\right) \mathrm{d}^{3} v_{\mathrm{A}} \mathrm{d}^{3} v_{\mathrm{B}}}{\int f_{2}\left(\boldsymbol{r}, \boldsymbol{v}_{\mathrm{A}}, \boldsymbol{v}_{\mathrm{B}}\right) \mathrm{d}^{3} v_{\mathrm{A}} \mathrm{d}^{3} v_{\mathrm{B}}} \\
=\frac{\int f_{2}\left(\boldsymbol{r}, \boldsymbol{v}_{\mathrm{A}}, \boldsymbol{v}_{\mathrm{B}}\right) \delta_{\mathrm{D}}^{(1)}\left(w_{\|}-v_{\mathrm{B} \|}+v_{\mathrm{A} \|}\right) \mathrm{d}^{3} v_{\mathrm{A}} \mathrm{d}^{3} v_{\mathrm{B}}}{\bar{n}^{2}[1+\xi(r)]}
\end{gathered}
$$

and finally obtain

$$
\begin{aligned}
1+\xi_{\mathrm{s}}\left(s_{\perp}, s_{\|}\right) & =\int_{-\infty}^{+\infty}[1+\xi(r)] \mathcal{P}_{w_{\|}}\left(w_{\|} \mid \boldsymbol{r}\right) \mathrm{d} w_{\|} \\
& =\int_{-\infty}^{+\infty}[1+\xi(r)] \mathcal{P}_{w_{\|}}\left(s_{\|}-r_{\|} \mid \boldsymbol{r}\right) \mathrm{d} r_{\|},
\end{aligned}
$$

which coincides with equation (4). The moment-generating function of the random variable $w_{\|}$is

$M_{w_{\|}}(t)=\frac{\int e^{t\left(v_{\mathrm{B} \|}-v_{\mathrm{A} \|}\right)} f_{2}\left(\boldsymbol{r}, \boldsymbol{v}_{\mathrm{A}}, \boldsymbol{v}_{\mathrm{B}}\right) \mathrm{d}^{3} v_{\mathrm{A}} \mathrm{d}^{3} v_{\mathrm{B}}}{\bar{n}^{2}[1+\xi(r)]}$.

Let us recap what we have done and achieved so far. Following a particle-based description and making use of the reduced distribution functions in phase-space, we have demonstrated that the streaming model is exact in the distantobserver approximation (and under the assumption of statistical homogeneity and isotropy in real space) provided that equation (13) is used to define $\boldsymbol{P}_{\boldsymbol{w}_{\|}}$or, equivalently, equation (15) is used to calculate the moment-generating function. It is worth stressing that our particle-based approach is completely rigorous also in multi-stream regions (where particles with different velocities are present at the same spatial location $\boldsymbol{x}$ ) and fully accounts for density-velocity correlations.

Alternative derivations of the streaming equation have been presented by other authors adopting more restrictive assumptions. They are generally based on a macroscopic description obtained by coarse graining $\hat{f}_{1}(\boldsymbol{x}, \boldsymbol{v})$ either in space (over patches of intermediate size between the typical interparticle separation and the cosmological scales of interest) or in time so that to erase discreteness effects and deal with a smooth function. Formally, a perfectly smooth distribution function, $\bar{f}_{1}(\boldsymbol{x}, \boldsymbol{v})$, is obtained by taking the RostokerRosenbluth fluid limit (Rostoker \& Rosenbluth 1960), i.e. by simultaneously letting $N \rightarrow \infty$ and the particle mass $m \rightarrow 0$ so that $\mathrm{Nm}=$ constant. This provides an approximated description of the system which is accurate until discreteness effects (particle collisions and correlations) cannot be neglected any longer. In fact, the microscopic, $n$-particle, and macroscopic densities are distinct quantities that evolve very differently: $\hat{f}_{1}$ is exact and satisfies the Klimontovic equation, the $n$-particle densities, $f_{n}$, (which are exact ensemble averages) are solutions of the BBGKY hierarchy, while $\bar{f}_{1}$ is an approximation and fulfills the Vlasov equation. Equipped with these definitions, we are now ready to compare our results with previous derivations of the streaming equation. Scoccimarro (2004) used the language of statistical field theory and characterized the matter content of the universe (in the single-stream regime) in terms of two continuous fields describing the density contrast $\delta(\mathbf{x})$ and the peculiar velocity $\boldsymbol{u}(\mathbf{x})$. In our notation, this is equivalent to assuming that $\bar{f}_{1}(\mathbf{x}, \boldsymbol{v})=\bar{n}[1+\delta(\mathbf{x})] \delta_{\mathrm{D}}^{(3)}[\boldsymbol{v}-\boldsymbol{u}(\mathbf{x})]$. Correlation functions were then computed by taking ensemble averages of the product of fields evaluated at different spatial locations. This method has been generalised to multi-streaming by Uhlemann et al. (2015) and Agrawal et al. (2017) (see also Seljak \& McDonald 2011), who wrote $\bar{n}[1+\delta(\mathbf{x})]=\int \bar{f}_{1}(\mathbf{x}, \boldsymbol{v}) \mathrm{d}^{3} v$ before computing the correlation function. In all cases, the authors were able to derive equation (4). However, since they rely on the collisionless fluid limit, all these approaches give a different velocity $\mathrm{PDF}$ and moment-generating function than our equations (13) and (15). In fact, our $f_{2}\left(\boldsymbol{r}, \boldsymbol{v}_{\mathrm{A}}, \boldsymbol{v}_{\mathrm{B}}\right)$ is replaced by the product $\bar{f}_{1}\left(\boldsymbol{x}_{\mathrm{A}}, \boldsymbol{v}_{\mathrm{A}}\right) \bar{f}_{1}\left(\boldsymbol{x}_{\mathrm{B}}, \boldsymbol{v}_{\mathrm{B}}\right)$ which completely neglects velocity correlations. While this is certainly an extremely good approximation for particle dark matter, it does not hold true in general. Note that our derivation is exact and applies to any system of particles (e.g. galaxies or their 
host haloes) without making assumptions regarding their interactions.

\subsubsection{Symmetry under particle exchange}

By construction, the velocity PDF in equation (13) is symmetric under particle exchange $(\mathrm{A} \leftrightarrow \mathrm{B})$ or parity transformations, i.e.

$\mathcal{P}_{w_{\|}}\left(w_{\|} \mid \boldsymbol{r}\right)=\mathcal{P}_{w_{\|}}\left(-w_{\|} \mid-\boldsymbol{r}\right)$.

In fact, the ordered pairs that can be formed with two particles equally contribute to $\left(w_{\|}, \boldsymbol{r}\right)$ and $\left(-w_{\|},-\boldsymbol{r}\right)$.

In order to visualise the velocity PDF for the DM particles of our $\mathrm{N}$-body simulation, we build an estimator for $\mathcal{P}_{w_{\|}}$by replacing the ensemble average in the definition of $f_{2}$ with an average over the simulation box (accounting for periodic boundary conditions). To speed the calculation up, we randomly sample $256^{3}$ particles and consider all the ordered pairs between them. Each pair is classified in $1 \times 1$ $\left(h^{-1} \mathrm{Mpc}\right)^{2}$ bins based on the values for $r_{\|}$and $r_{\perp}$. Finally, we build an histogram for $w_{\|}$in each bin. An example is shown in Fig. 2 where we examine real-space separations of $\pm r_{\|} \in[7,8) h^{-1} \mathrm{Mpc}$ and $r_{\perp} \in[0,1) h^{-1}$ Mpc. In each branch, the distribution clearly shows strongly asymmetric exponential tails. For $r_{\|}>0$, it has a negative skew meaning that the particles in the pairs tend to approach each other. The mean and rms values are $-2.4 h^{-1} \mathrm{Mpc}$ and $5.2 h^{-1} \mathrm{Mpc}$, respectively, while the mode is very close to zero.

\subsection{The streaming model for unordered pairs}

Before moving to the main goal of our work, we briefly discuss an alternative formulation of the streaming equation which highlights an interesting feature of RSD.

$\mathrm{N}$-body simulations provide valuable insights about the pairwise-velocity PDF. In this branch of the literature (Efstathiou et al. 1988; Zurek et al. 1994; Fisher et al. 1994; Scoccimarro 2004; Tinker et al. 2006; Reid \& White 2011; Zu \& Weinberg 2013; Bianchi et al. 2015), it is customary to use the pairwise los velocity for unordered pairs,

$w_{\text {los }}=w_{\|} \frac{r_{\|}}{\left|r_{\|}\right|}=w_{\|} \operatorname{sgn}\left(r_{\|}\right)$,

whose sign encodes physical information about the relative projected motion. If the elements of a pair approach (recede from) each other along the los, then $w_{\text {los }}$ is negative (positive).

It is worth stressing that the PDF of $w_{\text {los }}$ can not be inserted into equation (4) without first making some changes. In this section, we clarify the issue and derive the basic equation of the streaming model for unordered pairs. By construction, $w_{\text {los }}$ does not depend on the order of the galaxy/particle pairs and

$\mathcal{P}_{w_{\text {los }}}\left(w_{\text {los }} \mid \mathbf{r}\right)=\mathcal{P}_{w_{\text {los }}}\left(w_{\text {los }} \mid-\mathbf{r}\right)=\mathcal{P}_{w_{\text {los }}}\left(w_{\text {los }}\left|r_{\perp},\right| r_{\|} \mid\right)$.

For a generic $x$,

$\mathcal{P}_{w_{\|}}(x \mid \boldsymbol{r})= \begin{cases}\mathcal{P}_{w_{\text {los }}}\left(x\left|r_{\perp},\right| r_{\|} \mid\right) & \text {if } r_{\|} \geq 0, \\ \mathcal{P}_{w_{\text {los }}}\left(-x\left|r_{\perp},\right| r_{\|} \mid\right) & \text {if } r_{\|}<0,\end{cases}$

i.e. $\mathcal{P}_{w_{\text {los }}}$ coincides with the positive $r_{\|}$branch of $\mathcal{P}_{w_{\|}}$. Substituting in equation (4) and breaking the integral into two

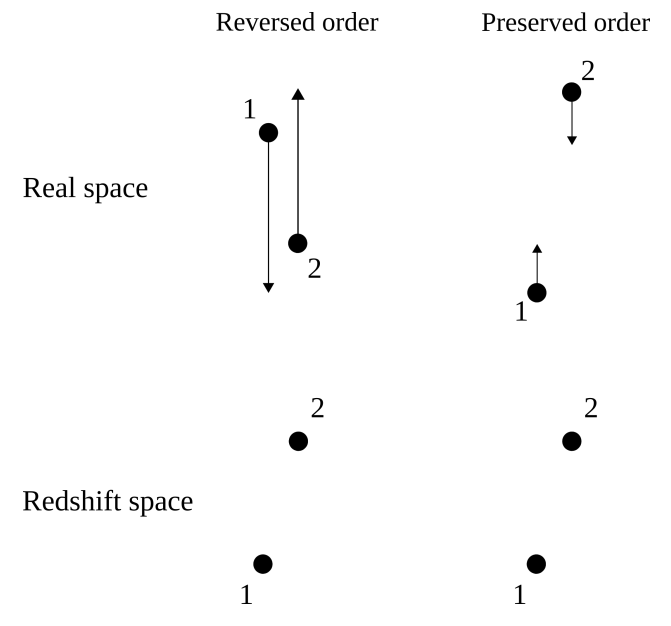

Figure 3. Schematic diagram illustrating how large relative infall velocities can reverse the order of a pair between real and redshift space (left-hand panel). The vertical arrows represent the los component of the individual velocities (assumed equal for simplicity). For comparison, the right-hand panel, shows a pair that preserves its order.

parts running over the positive and negative values of $r_{\|}$ respectively, we obtain

$1+\xi_{\mathrm{s}}\left(s_{\perp}, s_{\|}\right)=\int_{0}^{+\infty}[1+\xi(r)] g\left(s_{\|},\left|r_{\|}\right|, r_{\perp}\right) \mathrm{d}\left|r_{\|}\right|$,

with

$g\left(s_{\|},\left|r_{\|}\right|, r_{\perp}\right)=\mathcal{P}_{w_{\text {los }}}\left(-s_{\|}-\left|r_{\|}\right|\left|r_{\perp},\right| r_{\|} \mid\right)+\mathcal{P}_{w_{\text {los }}}\left(s_{\|}-\left|r_{\|}\right|\left|r_{\perp},\right| r_{\|} \mid\right)$.

This is the correct equation of the streaming model for unordered pairs. It can be re-written in compact form using the signed $r_{\|}$,

$1+\xi_{\mathrm{s}}\left(s_{\perp}, s_{\|}\right)=\int_{-\infty}^{+\infty}\left[1+\xi_{\mathrm{r}}(r)\right] \mathcal{P}_{w_{\text {los }}}\left[\left(s_{\|}-r_{\|}\right) \operatorname{sgn}\left(r_{\|}\right) \mid \mathbf{r}\right] \mathrm{d} r_{\|}$,

which allows a direct comparison with equation (14) for the ordered pairs. As we illustrate in Fig. 3, the first term on the right-hand side of equation (21) and the region with $r_{\|}<0$ in equation (22) refer to pairs that reverse their order between real and redshift space (i.e. having separation $s_{\|}>0$ in redshift space and $-\left|r_{\|}\right|<0$ in real space). On the other hand, the remaining terms are connected with pairs that preserve their ordering (i.e. both $s_{\|}$and $r_{\|}$are positive).

Pair reversal takes place more frequently at smaller realspace separations. The reason is twofold: i) smaller pairwise velocities are needed to swap the order of a pair when $r_{\|}$is small; ii) the distribution of $w_{\text {los }}$ shows a larger mean infall velocity and is more negatively skewed at small $r$. A detailed discussion on the impact of pair reversal on $\xi_{s}$ is presented in Appendix A.

\subsection{Applications of the streaming model}

\subsubsection{Pairwise velocities: mean infall and dispersion}

Most of the discussion on pairwise velocities focus on their low-order statistical moments. In our notation, the mean 
pairwise velocity is defined as

$\boldsymbol{v}_{12}(\mathbf{r})=\frac{\int\left(\boldsymbol{v}_{\mathrm{B}}-\boldsymbol{v}_{\mathrm{A}}\right) f_{2}\left(\boldsymbol{r}, \boldsymbol{v}_{\mathrm{A}}, \boldsymbol{v}_{\mathrm{B}}\right) \mathrm{d}^{3} v_{\mathrm{A}} \mathrm{d}^{3} v_{\mathrm{B}}}{\bar{n}^{2}[1+\xi(r)]}$,

which, in the literature, is often written in the single-stream fluid approximation as (e.g. Juszkiewicz et al. 1998; Reid \& White 2011)

$\boldsymbol{v}_{12}(\mathbf{r})=\frac{\left\langle\left(\boldsymbol{v}_{\mathrm{B}}-\boldsymbol{v}_{\mathrm{A}}\right)\left[1+\delta\left(\mathbf{x}_{\mathrm{A}}\right)\right]\left[1+\delta\left(\mathbf{x}_{\mathrm{B}}\right)\right]\right\rangle}{1+\xi(r)}$.

By symmetry, $\boldsymbol{v}_{12}(\mathbf{r})=v_{12}(r) \mathbf{r} / r$ and the sign of $v_{12}(r)$ indicates whether the elements of a pair, on average, approach each other or not. For a set of self-gravitating particles, $v_{12}(r)$ regulates the growth of the 2-point correlation function through the pair-conservation equation that derives from the BBGKY hierarchy (Davis \& Peebles 1977).

Similarly, the second moment of the pairwise velocities defines a tensor of components,

$\Sigma_{i j}(\boldsymbol{r})=\frac{\int \Delta_{i} \Delta_{j} f_{2}\left(\boldsymbol{r}, \boldsymbol{v}_{\mathrm{A}}, \boldsymbol{v}_{\mathrm{B}}\right) \mathrm{d}^{3} v_{\mathrm{A}} \mathrm{d}^{3} v_{\mathrm{B}}}{\bar{n}^{2}[1+\xi(r)]}$,

where $\Delta_{i}=\left(\boldsymbol{v}_{\mathrm{B}}-\boldsymbol{v}_{\mathrm{A}}\right) \cdot \hat{\boldsymbol{x}}_{\boldsymbol{i}}$ denotes the Cartesian components of the pairwise velocity vectors with respect to a right-handed orthonormal basis $\hat{\boldsymbol{x}}_{i}$. By symmetry, $\Sigma_{i j}$ can be expressed in terms of a few scalar functions

$\Sigma_{i j}(\boldsymbol{r})=\frac{\left[\Xi(r)+\frac{2}{3} \sigma_{v}^{2}\right] \delta_{i j}+[\Pi(r)-\Xi(r)] \frac{r_{i} r_{j}}{r^{2}}}{1+\xi(r)}$,

where $\sigma_{v}^{2}=\int v^{2} f_{1} \mathrm{~d}^{3} v / \int f_{1} \mathrm{~d}^{3} v$ denotes the mean square value of the peculiar particle velocity while $\Pi(r)$ and $\Xi(r)$ represent the contributions of correlations for the velocity components along $\hat{\boldsymbol{r}}$ and in the transverse directions, respectively (Davis \& Peebles 1977). The second moment of the pairwise-velocity component along the separation vector is

$\Sigma_{i j}(\boldsymbol{r}) \hat{r}_{i} \hat{r}_{j}=\frac{(2 / 3) \sigma_{v}^{2}+\Pi(r)}{1+\xi(r)}$,

while along any direction perpendicular to it $(\hat{\boldsymbol{t}} \cdot \hat{\boldsymbol{r}}=0)$

$\Sigma_{i j}(\boldsymbol{r}) \hat{t}_{i} \hat{t}_{j}=\frac{(2 / 3) \sigma_{v}^{2}+\Xi(r)}{1+\xi(r)}$.

The second moment tensor is isotropic if $\Pi(r)=\Xi(r)$. When this is not the case, the velocity dispersion along the line of sight depends on the pair orientation with respect to it (see Section 5.2 for further details).

\subsubsection{Some historical remarks}

Since the advent of galaxy redshift surveys, the streaming model has been a key tool to interpret clustering data. As we briefly mentioned in the introduction, in its early applications, it was used to make inferences about galaxy motions which are otherwise difficult to probe. The anisotropies in $\xi_{\mathrm{S}}\left(s_{\perp}, s_{\|}\right)$at small $s_{\perp}$ were translated into a typical pairwise velocity dispersion, $\sigma_{12}$, by assuming that (Peebles 1976, 1979)

$\mathcal{P}_{w_{\|}}\left(w_{\|} \mid \boldsymbol{r}\right)=\frac{1}{\sqrt{2} \sigma_{12}} \exp \left(-\frac{\sqrt{2}\left|w_{\|}\right|}{\sigma_{12}}\right)$, independently on the real-space separation. Quoting Peebles (1976), the expression above was 'meant only as a simple fitting function with one free parameter'. It was actually motivated by theoretical considerations and early $N$-body simulations showing that, on the spatial separations of interest: (i) the velocity PDF should be only weakly scale dependent, (ii) the second-moment tensor should be approximately isotropic and (iii) the pairwise velocity dispersion should be significantly larger than the mean. The small data sets available at the time (containing a few hundred galaxy redshifts) were found to be consistent with these assumptions.

As samples grew bigger and the analysis was extended to larger $s_{\perp}$, it was no longer possible to neglect the so-called 'streaming motions' i.e. the fact that the average relative velocity between galaxy pairs at fixed spatial separation does not vanish. If clustering is stable on small scales (i.e. galaxy groups are in virial equilibrium), then $v_{12}(r)=-H r$. On the other hand, for large separations, it is expected that $v_{12}(r) \rightarrow 0$. In between these asymptotic regimes, $v_{12}(r)<$ 0 and a net gravitational infall should be observed. As a natural generalization of equation (29), several authors then assumed that

$\mathcal{P}_{w_{\|}}\left(w_{\|} \mid \boldsymbol{r}\right)=\frac{1}{\sqrt{2} \sigma_{12}} \exp \left[-\frac{\sqrt{2}\left|w_{\|}-\bar{w}_{\|}(\boldsymbol{r})\right|}{\sigma_{12}}\right]$,

where $\bar{w}_{\|}(\boldsymbol{r})=\boldsymbol{v}_{12}(\mathbf{r}) \cdot \hat{z}=v_{12}(r) \hat{\boldsymbol{r}} \cdot \hat{z}$ denotes the line-ofsight component of the mean pairwise velocity and $v_{12}(r)$ is written as a function of $\xi(r)$ using approximate solutions to the pair-conservation equation calibrated against $N$-body simulations (Davis \& Peebles 1983; Bean et al. 1983; HaleSutton et al. 1989; Mo et al. 1993; Fisher et al. 1994; Zurek et al. 1994; Marzke et al. 1995; Loveday et al. 1996; Shepherd et al. 1997; Guzzo et al. 1997; Jing et al. 2002; Zehavi et al. 2002; Li et al. 2007).

The larger volumes covered by the current generation of redshift surveys lead to much more accurate measurements of galaxy clustering thus providing strong motivation for better models. Building upon the work by Fisher (1995), Reid \& White (2011) proposed a Gaussian approximation for $\mathcal{P}_{w_{\|}}\left(w_{\|} \mid \boldsymbol{r}\right)$ where the mean $v_{12}(r) \hat{\boldsymbol{r}} \cdot \hat{z}$ and the scaledependent dispersion $\sigma_{12}(\boldsymbol{r})$ are computed using standard perturbation theory by assuming that haloes are linearly biased tracers of the matter. At the same time, the realspace correlation function of the galaxies is evaluated using Lagrangian perturbation theory and also including higherorder bias terms. Finally, in order to account for the incoherent motion of galaxies within their host haloes (and also to mitigate the imperfections of perturbative calculations), the actual variance of the Gaussian PDF is written as $\sigma^{2}(\boldsymbol{r})=\sigma_{12}^{2}(\boldsymbol{r})+\sigma_{\mathrm{FoG}}^{2}$ with $\sigma_{\mathrm{FoG}}^{2}$ a nuisance parameter over which one needs to marginalize. The GSM has been later extended to use velocity statistics for biased tracers computed within the Convolution Lagrangian Perturbation Theory (CLPT, Wang et al. 2014) and the Convolution Lagrangian Effective Field Theory (CLEFT, Vlah et al. 2016). In its different versions, the GSM has been extensively applied to redshift surveys (Reid et al. 2012; Samushia et al. 2014; Alam et al. 2015; Chuang et al. 2017; Satpathy et al. 2017). 


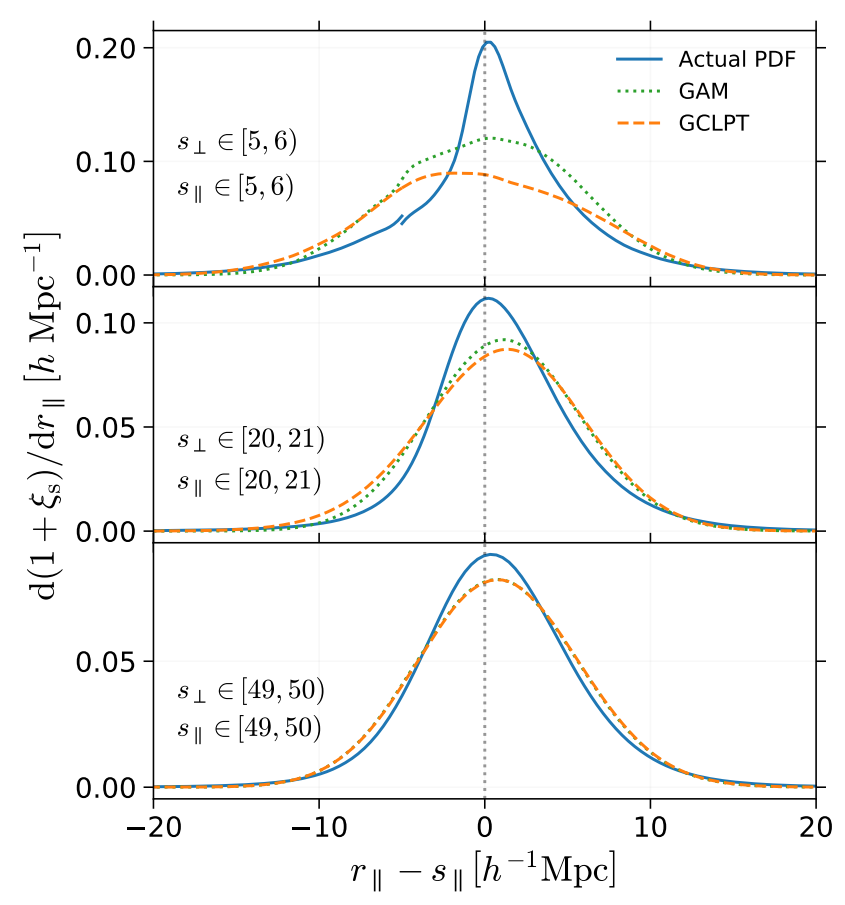

Figure 4. The integrand on the rhs of equation (4) is plotted as a function of $r_{\|}-s_{\|}$for three redshift-space separation vectors (whose radial and transverse components are given in units of $\left.h^{-1} \mathrm{Mpc}\right)$. The bottom panel refers to scales that are usually treated perturbatively, the middle panel to intermediate scales, while the top panel considers separations in the fully nonperturbative regime. In each case, we show three curves corresponding to different assumptions for the PDF of pairwise velocities. The solid curve corresponds to the actual data for the DMparticle pairs in the W0 simulation at $z=0$. The other curves are obtained by assuming a Gaussian PDF. For the dotted curve (GAM), the scale-dependent mean and variance are measured from the simulation while, for the dashed curve (GCLPT), they are estimated using CLPT. In all cases, we use the same realspace correlation function, $\xi(r)$, which has been measured from the $N$-body simulation.

\subsubsection{Limitations of the Gaussian approximation}

The GSM represents the state of the art in modelling RSD at the 2-point level. Considering DM haloes with masses between $10^{12}$ and $10^{13} h^{-1} \mathrm{M}_{\odot}$ at low redshift, Wang et al. (2014) showed that the two-point correlation $\xi_{\mathrm{s}}(\mathbf{s})$ predicted by the CLPT-based GSM agrees with $N$-body simulations to better than a few per cent for redshift-space separations larger than $\sim 20 h^{-1} \mathrm{Mpc}$ which are not too closely aligned with the line of sight (similar results are obtained for the CLEFT-based GSM, Vlah et al. 2016). The accuracy of the model degrades rapidly on smaller scales. We find similar trends when we study the correlation of DM particles, although, in this case, the model already departs from the simulations on substantially larger scales (see below for more quantitative information). It is instructive to investigate the origin of such behaviour and clarify the implications of the Gaussian ansatz for the pairwise-velocity PDF. To this goal, we focus on the DM particles in the W0 simulation at $z=0$ as they form a much larger sample than the DM haloes and are thus less affected by statistical noise. In Fig. 4, we plot the integrand appearing in the rhs of equation (4) as a func- tion of $r_{\|}-s_{\|}$for three redshift-space separation vectors and using three different 'models' for $\mathcal{P}_{\boldsymbol{w}_{\|}}\left(w_{\|} \mid \boldsymbol{r}\right)$. The solid line is obtained by measuring the velocity $\mathrm{PDF}$ at different pair separation vectors directly from the simulation. The dotted line (GAM) corresponds to assuming a Gaussian PDF with the actual scale-dependent mean and variance measured in the simulation. Finally, the dashed line (GCLPT) uses CLPT to predict the mean and variance (up to an additive constant calibrated using simulations, see Section 5 and Wang et al. (2014) for further details) of the Gaussian PDF. In all cases, we do not compute $\xi(r)$ perturbatively but we measure it directly from the simulation. The top panel shows that both the Gaussian ansatz for $\mathcal{P}_{w_{\|}}$and the CLPT calculations are very inaccurate at small scales. Integrating the different curves gives $\xi_{\mathrm{s}}=0.64$ for the $N$-body based PDF while we obtain $\xi_{\mathrm{s}}=0.70$ and 0.44 for the GAM and GCLPT models, respectively. The middle panel refers to a separation vector with components $s_{\|}=s_{\perp} \simeq 20.5 h^{-1} \mathrm{Mpc}$. In this case, pairs with $5 \lesssim r_{\|} \lesssim 35 h^{-1} \mathrm{Mpc}$ contribute to $\xi_{\text {s }}$ and those with $r_{\|} \simeq 20 h^{-1} \mathrm{Mpc}$ give the largest signal. Both Gaussian approximations, however, reach their maximum for slightly larger values of $r_{\|}$and give rise to less sharply peaked integrands in the streaming equation. Although the two curves based on the Gaussian approximations seem to be rather similar, once integrated, give rise to significantly different values of $\xi_{\mathrm{s}}$. We find $\xi_{\mathrm{s}}=0.049$ when the actual moments are used and $\xi_{\mathrm{s}}=0.063$ for the CLPT predictions. This difference mainly originates from the regions on the lhs from the peak, i.e. for $r_{\|}<s_{\|}$where perturbative calculations become less reliable. Note that the actual value of the redshiftspace correlation is $\xi_{\mathrm{s}}=0.048$ which nearly coincides with the best Gaussian approximation in spite of the fact that the corresponding integrals in Fig. 4 appear to be quite different. Even though we provided only one specific example here, we find that this serendipitous coincidence holds true for a broad range of redshift-space separation vectors $\mathbf{s}$. This result provides motivation for improving the GSM by combining it with enhanced estimates for the pairwise-velocity moments. However, the success of the GSM on these intermediate scales appears to be fortuitous as the model does not reproduce the correct shape of the integrand in the streaming equation. The bottom panel shows that the situation improves only slightly when we consider larger redshift-space separations. In this case, the CLPT calculations can be calibrated to accurately reproduce the first two moments of the pairwise velocities and no obvious difference can be noted between the GAM and GCLPT approximations. However, even at a separation of $s \simeq 70 h^{-1} \mathrm{Mpc}$, the Gaussian models for the velocity $\mathrm{PDF}$ cannot accurately reproduce the shape of the function $\mathrm{d}\left(1+\xi_{\mathrm{s}}\right) / \mathrm{d} r_{\|}$measured in the simulation. In spite of this, once the integral over $r_{\|}$is performed, they give values for $\xi_{\mathrm{s}}$ which are accurate at the few per cent level.

The aim of this paper is to introduce a more general fitting function for the pairwise-velocity PDF that, once inserted in the streaming model, provides more accurate results for the redshift-space correlation function than the GSM. The advantage is twofold: first, on large scales and in the era of precision cosmology, we would be able to make predictions that do not rely on fortuitous cancellations and, second, extending the accuracy of the streaming model to smaller spatial separations would allow us to probe modified 
gravity and interacting dark-energy models as mentioned in the introduction. Related work has been presented by Uhlemann et al. (2015) and Bianchi et al. (2015, 2016) who accounted for skewness in the PDF by using the Edgeworth expansion around a Gaussian probability density and by superposing multiple Gaussian (or quasi-Gaussian) distributions, respectively.

\section{STATISTICS OF PAIRWISE VELOCITIES}

The streaming model is formulated in terms of $w_{\|}$or $w_{\text {los }}$ while analytical calculations (perturbative or not) generally deal with the radial and transverse components of the pairwise velocities. In this section, we derive the relation between the cumulants of these different components. In order to provide some illustrative examples, we compute several velocity statistics for the DM particles and the haloes in the W0 simulation at redshift $z=0$.

\subsection{Cumulants}

In Fig. 5, we investigate how the first four normalised cumulants of $\mathcal{P}_{w_{\|}}$for the DM particles, depend on the real-space separation vector $\mathbf{r}$. Specifically, we consider the mean $\mu_{\|}=$ $\left\langle w_{\|}\right\rangle_{\mathrm{c}}$, the variance $\sigma_{\|}^{2}=\left\langle w_{\|}^{2}\right\rangle_{\mathrm{c}}$, the skewness $\gamma_{\|}=\left\langle w_{\|}^{3}\right\rangle_{\mathrm{c}} / \sigma_{\|}^{3}$ and the kurtosis $k_{\|}=\left\langle w_{\|}^{4}\right\rangle_{\mathrm{c}} / \sigma_{\|}^{4}$. As expected, the odd cumulants undergo parity inversion as the sign of $r_{\|}$changes while the even cumulants are parity invariant. Note that the cumulants of $\mathcal{P}_{w_{\text {los }}}$ can also be read from Fig. 5 by looking at the region with $r_{\|}>0$. In this case, the mean velocity and the skewness are always negative meaning that $\mathcal{P}_{w_{\text {los }}}$ is asymmetric as it is more likely to find infalling pairs at the scales we consider (see also Scoccimarro 2004). The velocity PDF is leptokurtic (i.e. $k>3$ ) meaning that it has a sharper peak and heavier tails compared to a Gaussian distribution. Although both $|\gamma|$ and $k$ decrease with increasing $r_{\|}$and $r_{\perp}, \mathcal{P}_{w_{\text {los }}}$ always differs substantially from a Gaussian probability density.

\subsection{Radial, transverse and los pairwise velocities}

The peculiar shape of the contour levels in Fig. 5 is mainly regulated by the angle that the pair separation forms with the los. This can be shown as follows. The pairwise velocity of an ordered pair, $\boldsymbol{v}_{2}-\boldsymbol{v}_{1}$, can be decomposed into radial (i.e. along the pair-separation vector) and transverse components:

$w_{\mathrm{r}}=\left(\boldsymbol{v}_{2}-\boldsymbol{v}_{1}\right) \cdot \hat{\boldsymbol{r}}$,

$\mathbf{w}_{\mathrm{t}}=\left(\boldsymbol{v}_{2}-\boldsymbol{v}_{1}\right)-w_{\mathrm{r}} \hat{\mathbf{r}}$.

In a homogenous and isotropic universe, the statistical properties of $w_{\mathrm{r}}$ and $\mathbf{w}_{\mathrm{t}}$ only depend on $r$. Introducing a preferential los direction, however, breaks the rotational invariance so that $\mathcal{P}_{w_{\|}}$depends on both $r_{\|}$and $r_{\perp}$. Let us consider the normal vector to the plane defined by the pair separation and the los,

$\boldsymbol{n}=\hat{\boldsymbol{r}} \times \hat{z}$, and form a right-handed orthonormal basis using $\hat{\mathbf{r}}, \hat{\mathbf{n}}$ and the additional unit vector $\hat{\mathbf{e}}$. Only the component of $\mathbf{w}_{\mathrm{t}}$ perpendicular to $\boldsymbol{n}$ (and thus parallel to $\hat{\mathbf{e}}$ ),

$\boldsymbol{w}_{\mathrm{e}}=\left(\boldsymbol{w}_{\mathrm{t}} \cdot \hat{\boldsymbol{e}}\right) \hat{\boldsymbol{e}}=\left(\boldsymbol{v}_{2}-\boldsymbol{v}_{1}\right)-w_{\mathrm{r}} \hat{\boldsymbol{r}}-\left(\mathbf{w}_{\mathrm{t}} \cdot \hat{\boldsymbol{n}}\right) \hat{\boldsymbol{n}}$,

contributes to $w_{\|}$. By defining the angle $\theta$ so that $\cos \theta=\hat{\mathbf{r}}$. $\hat{z}=r_{\|} / r$, it follows that $\hat{\mathbf{e}} \cdot \hat{z}= \pm \sin \theta$ depending on the relative orientation of the pair separation and the los. Eventually, we can write

$w_{\|}=w_{\mathrm{r}} \cos \theta+w_{\mathrm{p}} \sin \theta$,

with

$w_{\mathrm{p}}=\operatorname{sgn}(\hat{\boldsymbol{e}} \cdot \hat{\boldsymbol{z}}) w_{\mathrm{e}}$.

The cumulants of $w_{\|}$at fixed $r$ and $\theta$ can then be expressed in terms of the cumulants and cross-cumulants of $w_{\mathrm{r}}$ and $w_{\mathrm{p}}$ at fixed $r$. It follows from equation (35) that

$\left\langle w_{\|}^{n}\right\rangle_{\mathrm{c}}=\sum_{i_{1}=1}^{2} \sum_{i_{2}=1}^{2} \ldots \sum_{i_{n}=1}^{2}\left\langle w_{i_{1}} w_{i_{2}} \ldots w_{i_{n}}\right\rangle_{\mathrm{c}}$,

where $w_{1} \equiv w_{\mathrm{r}} \cos \theta$ and $w_{2} \equiv w_{\mathrm{p}} \sin \theta$. Terms involving odd powers of $w_{\mathrm{p}}$ vanish due to statistical isotropy (i.e. the probability distribution of $w_{\mathrm{p}}$ is symmetric with respect to zero, reflecting the fact that all orientations of $\hat{\boldsymbol{r}}$ with respect to $\hat{z}$ are equally likely). For the normalised cumulants shown in Fig. 5, we obtain:

$\mu_{\|}=\mu_{\mathrm{r}} \cos \theta$,

$\sigma_{\|}^{2}=\sigma_{\mathrm{r}}^{2} \cos ^{2} \theta+\sigma_{\mathrm{p}}^{2} \sin ^{2} \theta$,

$\gamma_{\|}=\sigma_{\|}^{-3}\left[\left\langle w_{\mathrm{r}}^{3}\right\rangle_{\mathrm{c}} \cos ^{2} \theta+3\left\langle w_{\mathrm{r}} w_{\mathrm{p}}^{2}\right\rangle_{\mathrm{c}} \sin ^{2} \theta\right] \cos \theta$,

$k_{\|}=\sigma_{\|}^{-4}\left\{\left\langle w_{\mathrm{r}}^{4}\right\rangle_{\mathrm{c}} \cos ^{4} \theta+\left\langle w_{\mathrm{p}}^{4}\right\rangle_{\mathrm{c}} \sin ^{4} \theta+\right.$

$$
\left.6\left[\left\langle w_{\mathrm{r}}^{2} w_{\mathrm{p}}^{2}\right\rangle_{\mathrm{c}}-2 \mu_{\mathrm{r}}\left\langle w_{\mathrm{r}} w_{\mathrm{p}}^{2}\right\rangle_{\mathrm{c}}\right] \cos ^{2} \theta \sin ^{2} \theta\right\} .
$$

\subsubsection{DM particles}

In the left column of Fig. 6, we show the radial dependence of the different isotropic terms appearing in the equations above for the particles in the final snapshot $(z=0)$ of the W0 simulation. In the top row, we compare $\mu_{\mathrm{r}}$ measured in our simulation with the predictions of linear and oneloop Convolution Lagrangian Perturbation Theory (CLPT Carlson et al. 2013; Wang et al. 2014). In the second row, we plot $\sigma_{\mathrm{r}}^{2}$ and $\sigma_{\mathrm{p}}^{2}$ for the $N$-body particles and contrast them with the corresponding CLPT results at one loop (after shifting them vertically in order to match the simulation output at $r=100 h^{-1} \mathrm{Mpc}$ ). The figures indicate that state-of-the-art perturbative approaches qualitatively reproduce the scale dependence of the lowest-order cumulants for $r>20-30 h^{-1} \mathrm{Mpc}$. However, they provide accurate predictions only on much larger scales. Finally, the third and fourth rows present the various contributions to the skewness and kurtosis of $w_{\|}$. Note that the cross-cumulants of the correlated random variables $w_{\mathrm{r}}$ and $w_{\mathrm{p}}$ are sub-dominant but non-negligible.

\subsubsection{DM haloes and pairwise velocity bias}

We now contrast the previous results with the isotropic velocity statistics for the DM haloes identified at $z=0$ in the 

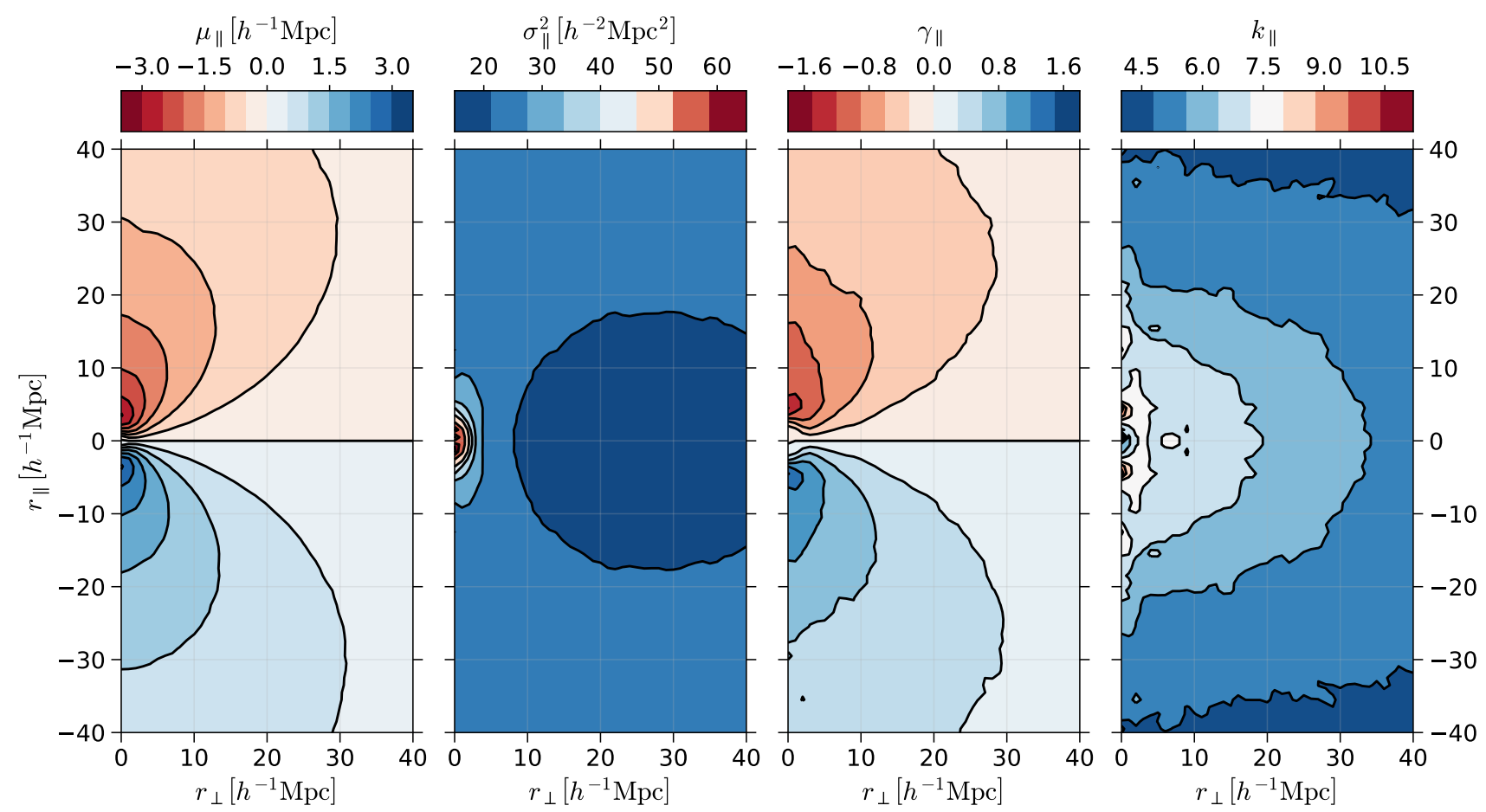

Figure 5. Normalised cumulants of the pairwise los velocity for ordered pairs of DM particles in the W0 simulation.

W0 simulation. In the middle column of Fig. 6, we show the cumulants and cross-cumulants for the radial and tangential pairwise velocities measured using the halo bulk velocities. It is evident that halo pairs (that trace peaks in the density field) tend to approach each other at a slightly greater velocity than particle pairs (that also populate underdense regions where the velocity divergence is positive). Haloes have also smaller velocity dispersions, i.e. they trace a colder velocity field than the DM (see also Carlberg \& Couchman 1989; Couchman \& Carlberg 1992; Cen \& Ostriker 1992; Gelb \& Bertschinger 1994; Evrard et al. 1994; Summers et al. 1995; Colín et al. 2000). Following Carlberg (1994), we introduce the pairwise velocity bias as the ratio between the halo and DM rms pairwise velocities. Its variation with the pair separation is shown in Fig. 7 for the radial and the tangential components of the velocity. In both cases, the 'antibias' assumes values $\sim 0.8$ on large scales and becomes very strong for $r<15 h^{-1}$ Mpc. Fig. 6 shows that also the higher-order cumulants for the haloes depart from those for the DM, especially at small separations. This reflects the fact that the shape of the pairwise velocity PDF is different for DM particles and haloes.

In the right column of Fig. 6, we investigate the dependence of the velocity cumulants on halo mass. More massive objects show larger mean infall velocities, dispersions, and fourth-order cumulants. On the other hand, we could not detect any mass dependence for the third-order cumulant. The latter, however, is always negative indicating the the halo velocity PDF is asymmetric (and thus non Gaussian) for all the separations and masses considered here. The amplitude of the fourth cumulant (invariably larger than 3) also shows that the PDF is leptokurtic with heavy tails.

\subsection{Dissecting the pairwise-velocity distribution}

The pairwise-velocity distribution of DM particles is shaped by the highly non-linear physics of gravitational instability and, for this reason, is very difficult to model analytically starting from first principles. A simplified approach relies on a phenomenological description that exploits the internal dynamics of DM haloes (e.g Sheth 1996; Sheth \& Diaferio 2001; Tinker 2007). To provide further insight into the importance of virialised structures, we investigate the halo contribution to $\mathcal{P}_{w_{\text {los }}}$. We first classify the DM particles in our simulation according to whether they belong to haloes or to the field. We then partition $\mathcal{P}_{w_{\text {los }}}$ into the contributions of halo-halo, halo-field and field-field pairs. Note that the concept of 'field particle' is not absolute as it depends on the mass resolution of the simulation and the minimum halo mass which is considered. In the W0 simulation, we classify 69.3 per cent of the particles as belonging to the field at $z=0$. If we were resolving haloes with a mass $M<1.2 \times 10^{13} h^{-1} \mathrm{M}_{\odot}$, then the fraction of field particles would decrease. Basically, our 'halo particles' account for the matter content of galaxy groups and clusters of galaxies. We show a few examples in Fig. 8. For real-space separations that are smaller than the typical halo size, $r<1 h^{-1} \mathrm{Mpc}$, halo-halo pairs give the dominant contribution to $\mathcal{P}_{w_{\text {los }}}\left(w_{\text {los }} \mid \mathbf{r}\right)$ for all $w_{\text {los }}$ whereas the field-field pairs only matter at very low $w_{\text {los }}$. For $r>1 h^{-1} \mathrm{Mpc}$, instead, the wings of $\mathcal{P}_{w_{\text {los }}}$ are regulated by the halo-halo pairs while the core of the PDF is determined by the field-field pairs. Halo-field pairs are always subdominant. The fieldfield term peaks at $w_{\text {los }} \simeq 0$, is negatively skewed (although it becomes practically symmetric for $r_{\|}<1 h^{-1} \mathrm{Mpc}$ ) and shows rapidly decaying exponential wings. Halo-halo pairs are characterized by larger mean infall velocities and velocity dispersions with respect to their field-field counterparts. 


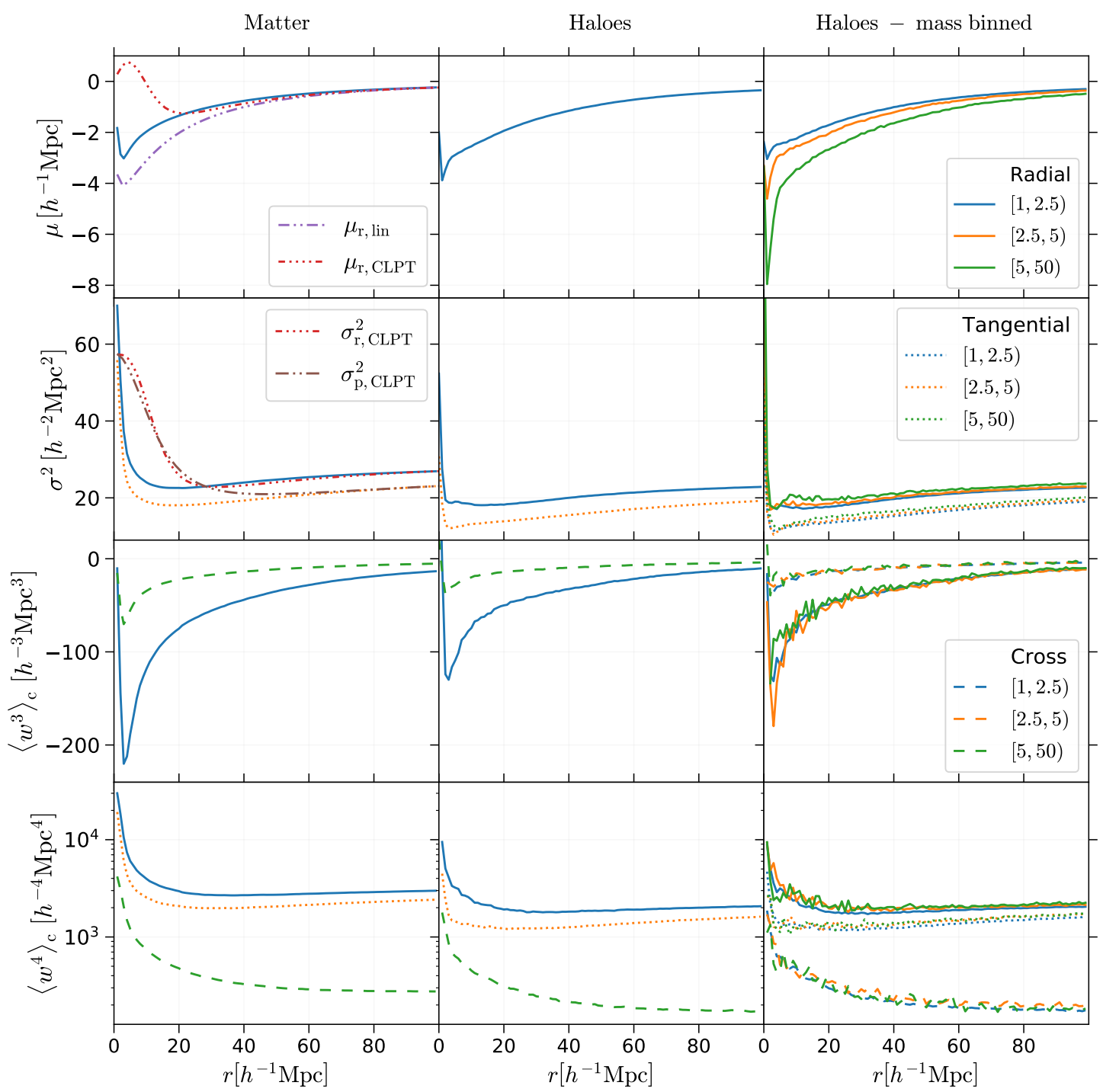

Figure 6. Cumulants and cross-cumulants of the radial and tangential pairwise velocities contributing to the first 4 normalised cumulants of $w_{\|}$, see equations (38) - (41). The solid, dotted and dashed lines represent the radial, tangential and cross components, respectively. In the left column, all quantities have been measured from the velocities of DM particles in the W0 simulation at $z=0$. One-loop (and linear, for $\mu_{\mathrm{r}}$ ) CLPT predictions are also shown in the two topmost panels. A constant has been added to the CLPT results for $\sigma_{\mathrm{r}}^{2}$ and $\sigma_{\mathrm{p}}^{2}$ in order to match the simulation output at $r=100 h^{-1} \mathrm{Mpc}$. The middle column, shows the results for the bulk velocities of the DM haloes identified in the same simulation snapshot. Finally, the dependence of the results on the halo mass is investigated in the right column using different mass intervals indicated in units of $10^{13} h^{-1} \mathrm{M}_{\odot}$.

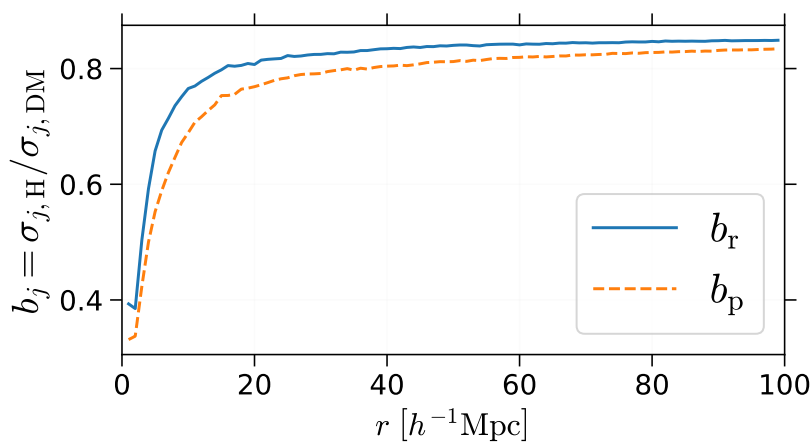

Figure 7. Pairwise-velocity bias for radial (solid) and tangential (dashed) motion.
The exponential tails of their negatively skewed distribution are also much fatter. In $\xi_{\mathrm{s}}$, halo-halo pairs produce the FoG enhancement at small $s_{\perp}$, while field-field pairs completely dominate the signal at large $s$.

In Fig. 9, we further partition the halo-halo pairs into subsamples based on the halo mass (for simplicity, cross pairs formed by particles in different halo-mass bins are not considered here). This procedure reveals that haloes of different masses $^{2}$ are characterized by very different pairwisevelocity distributions. Not only the velocity dispersion increases with the halo mass but also the mean infall velocity

2 We also considered additional variables as the halo spin parameter and triaxiality but their impact on $\mathcal{P}_{w_{\text {los }}}$ was too small to be isolated. 


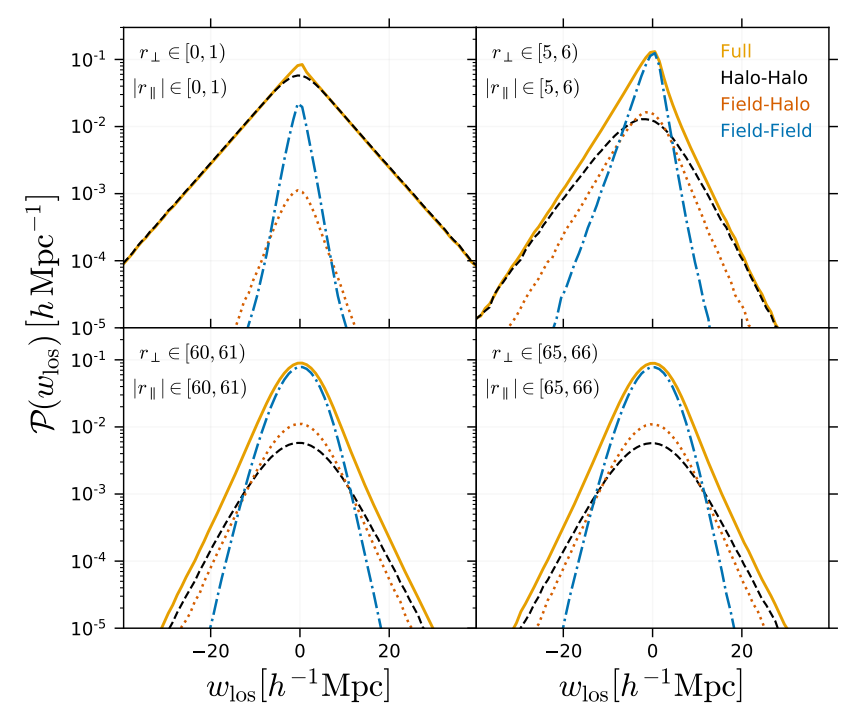

Figure 8. The los pairwise-velocity distribution of the particles in the W0 simulation at $z=0$ is decomposed into simpler elements. The figure shows the contributions of halo-halo, field-halo and field-field pairs for six different real-space separation vectors (expressed in $h^{-1} \mathrm{Mpc}$ ).

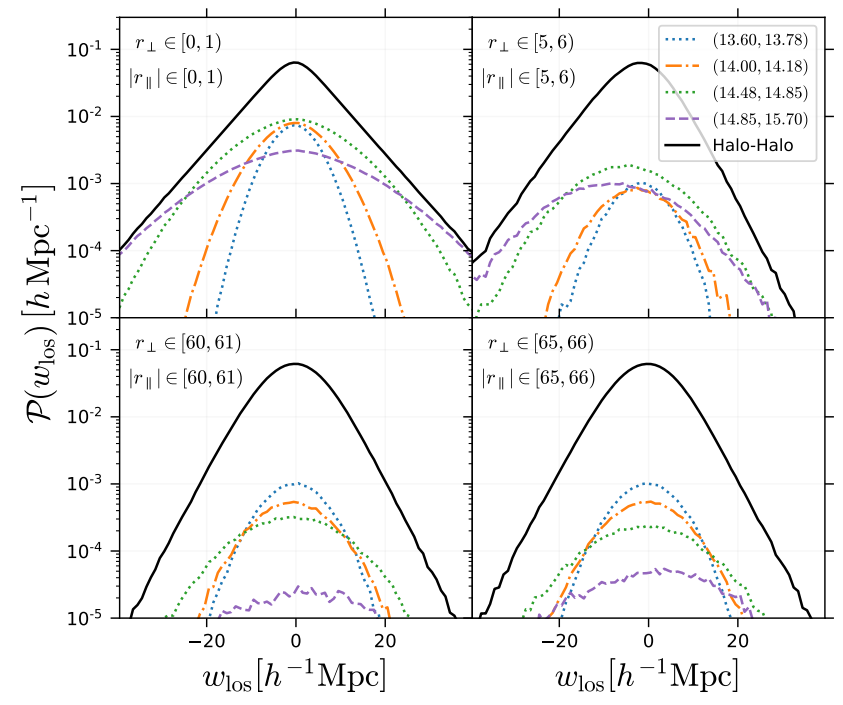

Figure 9. The los pairwise-velocity distribution of the halo-halo term is further partitioned into the contributions of various halo log-mass bins (the bin boundaries in $\log _{10}\left[M_{\mathrm{vir}} /\left(h^{-1} \mathrm{M}_{\odot}\right)\right]$ are indicated in the labels) for different real-space separation vectors (expressed in $h^{-1} \mathrm{Mpc}$ ). For simplicity, only 'diagonal' terms in which both particles reside in haloes of the same bin are shown.

grows in magnitude. The skewness can even reverse sign for cluster-sized haloes. All this shows the complexity behind the overall distribution of $w_{\text {los }}$ and clarifies why the PDF is so difficult to model accurately.

\section{A NEW FITTING FUNCTION}

It has been proposed that $\mathcal{P}_{w_{\text {los }}}\left(w_{\text {los }} \mid \mathbf{r}\right)$ can be more easily described as a superposition of simpler elementary functions.
The pairwise peculiar velocity dispersion depends on the (suitably defined) local density of the pairs (Kepner et al. 1997) and its PDF can be modelled as a weighted sum of basic Gaussian terms evaluated at fixed halo masses and environment densities (Sheth 1996; Sheth \& Diaferio 2001; Tinker et al. 2006; Tinker 2007). Integrating out the degrees of freedom due to the haloes, $\mathcal{P}_{w_{\text {los }}}\left(w_{\text {los }} \mid \mathbf{r}\right)$ can be approximated as the superposition of univariate Gaussian (Bianchi et al. 2015) or quasi-Gaussian (Bianchi et al. 2016) distributions whose cumulants are drawn from a multivariate Gaussian distribution.

Although relatively new in the field of cosmology, similar techniques have actually been in use for over a century in statistics, finance and the theory of turbulence. The basic idea is to suitably combine uncountably many elements of a parametric family of PDFs to model heavy tailed and skewed distributions. In statistics, an (uncountable) 'mixture' or 'compound' probability distribution is defined by the relation

$\mathcal{P}_{x}(x \mid \tau)=\int \mathcal{P}_{x}(x \mid \mathbf{f}) \mathcal{P}_{\mathbf{f}}(\mathbf{f} \mid \tau) \mathrm{d}^{n} f=\mathcal{P}_{x} \circ \mathcal{P}_{\mathbf{f}}$,

where $x$ denotes the random variable of interest (subject to the condition $\tau$ ) and $\mathbf{f}$ is a $n$-dimensional array containing the factors (or latent variables) that influence the distribution of $x$. The function $\mathcal{P}_{x}(x \mid \mathbf{f})$ gives the conditional probability density of $x$ in a subpopulation with given $\mathbf{f}$ while the 'mixing distribution' $\mathcal{P}_{\mathbf{f}}$ is the joint probability density (or the statistical measure) of the factors. If $\mathcal{P}_{x}(x \mid \mathbf{f})$ is a Gaussian distribution, $\mathcal{N}_{x}\left(x ; \mu, \sigma^{2}\right)$, then the variable $x$ is called a mixture of Gaussians (or normals). Scale mixtures of normals (where mixing only involves $\sigma^{2}$ ) are widely used to model symmetric distributions with heavy tails. Location mixtures of normals (where mixing only involves $\mu$ ) are commonly used to model skewed ${ }^{3}$ distributions. Joint location and scale mixing gives rise to skewed distributions with heavy tails.

The above-mentioned models for $\mathcal{P}_{w_{\text {los }}}\left(w_{\text {los }} \mid \mathbf{r}\right)$ can be phrased in this language. For instance, Bianchi et al. (2015) assume that $w_{\text {los }}$ is a joint location and scale mixture of Gaussians with a bivariate Gaussian mixing distribution. In this case, the scatter in $\mu$ and $\sigma$ is meant to represent physical variability due to some latent (but not-so-well-specified) environmental density. This method compresses the information contained in $\mathcal{P}_{w_{\text {los }}}\left(w_{\text {los }} \mid \mathbf{r}\right)$ into five parameters (the mean values for $\mu$ and $\sigma$ and the three elements of their covariance matrix) that change smoothly with $\mathbf{r}$. Although the model matches well the outcome of numerical simulations on large scales, it has two drawbacks: i) by definition, $\sigma$ is non negative and thus cannot follow a Gaussian distribution ${ }^{4}$; ii) the resulting $\mathcal{P}_{w_{\text {los }}}\left(w_{\text {los }} \mid \mathbf{r}\right)$ cannot reproduce the large skewness measured at small separations $\left(r<5-10 h^{-1}\right.$ $\mathrm{Mpc}$ depending on the redshift) for particles and haloes in $N$-body simulations (Bianchi et al. 2016). For this reason, Bianchi et al. (2016) replace the mixture of Gaussians with a mixture of skewed quasi-Gaussians obtained by applying

3 Note, however, that a location mixture with Gaussian mixing distribution yields another Gaussian.

4 To address this issue, Bianchi et al. (2016) truncate the $\sigma$ distribution at zero and renormalise it to account for the probability in the Gaussian tail with negative support. 
the Edgeworth expansion to first order. However, in order to limit the number of degrees of freedom of the model, the skewness of $\mathcal{P}_{x}(x \mid \mathbf{f})$ is not used as a third factor over which the mixing is performed but is deterministically linked to the variances of $\sigma$ and $\mu$ by means of an ansatz that introduces an additional free parameter.

While these recent efforts have led to the development of tools that can closely approximate $\mathcal{P}_{w_{\text {los }}}\left(w_{\text {los }} \mid \mathbf{r}\right)$ on a wide range of scales, they are based on to a phenomenological description that provides little insight into the underlying physics. At the current stage of development, the models do not have predictive power. They essentially offer a language and a convenient class of fitting functions that can be used to describe the output of simulations with different gravity models and retrieve information on the cosmological parameters and the law of gravity from the observed $\xi_{\mathrm{s}}$ (assuming a functional form for the scale dependence of the model parameters). Their complexity, however, is growing rapidly and, as we discussed above, ad hoc assumptions are required to limit their degrees of freedom while extending their range of validity. Given this premise, we follow here a pragmatic and complementary approach by proposing a fitting function for $\mathcal{P}_{w_{\text {los }}}\left(w_{\text {los }} \mid \mathbf{r}\right)$ that closely reproduces the features seen in $N$-body simulations and provides an excellent fit to them at all scales. For this purpose, we search the statistics literature for a family of analytic PDFs with the following characteristics: i) unimodality; ii) presence of quasi-exponential tails; iii) highly tunable low-order cumulants (in particular skewness); iv) possibility of reducing to the Gaussian distribution in some limit. We end up selecting the generalised hyperbolic distribution (GHD) which will be precisely defined in the next section. As in Peebles (1976) and Reid \& White (2011), we do not give any physical motivation in support of our choice but we note that, interestingly enough, the GHD describes a particular mixture of normals.

\subsection{The generalised hyperbolic distribution}

\subsubsection{The inverse Gaussian distribution}

Let us consider a one-dimensional standard Wiener process with drift $v \in \mathbb{R}_{>0}$ and diffusion coefficient $\sigma \in \mathbb{R}_{>0}$. At time $t$, the position $x$ of a Brownian particle follows the distribution $\mathcal{N}_{x}\left(x ; v t, \sigma^{2} t\right)$. The first-passage time of the level $\ell>0$ by a Brownian walker is distributed $\mathrm{as}^{5}$ (Schrödinger 1915)

$f_{t}(t ; \ell, v, \sigma)=\frac{\ell}{\sqrt{2 \pi} \sigma} t^{-3 / 2} \exp \left[-\frac{(\ell-v t)^{2}}{2 \sigma^{2} t}\right]$.

When expressed in terms of the parameters $\mu=\ell / v$ and $\gamma=\ell^{2} / \sigma^{2}$, this equation defines the 'inverse Gaussian distribution' (see e.g. Chhikara \& Folks 1989; Seshadri 1999, for a comprehensive review of its properties),

$\mathcal{I}_{t}(t ; \mu, \gamma)=\left(\frac{\gamma}{2 \pi}\right)^{1 / 2} t^{-3 / 2} \exp \left[-\frac{\gamma(t-\mu)^{2}}{2 \mu^{2} t}\right]$.

5 In cosmology, this PDF has been used by Bond et al. (1991) to solve the cloud-in-cloud problem in the excursion-set model for the halo mass function.
This PDF provides a classic model for non-negative, unimodal and positively skewed data and is widely employed to perform lifetime and survival studies in ecology, engineering, finance, law and medicine. The mean of the distribution coincides with the location parameter $\mu$, while the variance $\left(\mu^{3} / \gamma\right)$, skewness $(3 \sqrt{\mu / \gamma})$ and kurtosis $(15 \mu / \gamma)$ also depend on the scale parameter $\gamma$. The name inverse Gaussian was coined by Tweedie (1956) and only refers to the fact that, while the Gaussian distribution describes the distribution of $x$ at fixed $t, I_{t}$ gives the PDF of the time at which the particles first cross a fixed position.

\subsubsection{Generalised inverse Gaussian distribution}

In the 1940s and 1950s, a larger family of unimodal PDFs with positive support was introduced. Since this class includes the inverse Gaussian distribution as a special case, it now goes under the name of the 'generalised inverse Gaussian distribution' (Jørgensen 1982). The corresponding PDF for the random variable $t>0$ is

$\mathcal{G}_{t}(t ; \lambda, \chi, \psi)=\frac{(\psi / \chi)^{\lambda / 2}}{2 K_{\lambda}(\sqrt{\psi \chi})} t^{\lambda-1} \exp \left[-\frac{1}{2}\left(\psi t+\frac{\chi}{t}\right)\right]$,

where $\lambda \in \mathbb{R}, \chi \in \mathbb{R}_{>0}, \psi \in \mathbb{R}_{>0}$ and $K_{\lambda}$ is the modified Bessel function of the second kind with order $\lambda$ (Abramowitz \& Stegun 1972). For $\lambda=-1 / 2, \psi=\gamma / \mu^{2}$ and $\chi=\gamma$, equation (45) reduces to the inverse Gaussian distribution given in equation (44). The generalised inverse Gaussian distribution can be interpreted as the distribution of the first passage time or, depending on the sign of $\lambda$, the last exit time of more complicated diffusion processes than Brownian motion (Vallois 1991). Note that, for large values of $t, \mathcal{G}_{t}$ has a thin tail $\propto t^{\lambda-1} \exp (-\psi t / 2)$. Another interesting property is that, if $t$ follows $\mathcal{G}_{t}(t ; \lambda, \chi, \psi)$, then $t^{-1}$ follows $\mathcal{G}_{t^{-1}}\left(t^{-1} ;-\lambda, \psi, \chi\right)$. In analogy with $\mathcal{I}_{t}$, the generalised inverse Gaussian distribution finds many direct applications in risk assessment and queue modelling. Moreover, it is commonly used as a mixing distribution whenever there is the need for skewed weighting. This practice was initiated by Sichel (1974) who used mixtures of Poisson distributions to model the distribution of sentence lengths and word frequencies.

\subsubsection{Normal variance-mean mixtures}

Let us return to the Wiener process with drift we introduced in Section 6.1.1. This time, however, we assume that the Brownian particles start from $x=\alpha \in \mathbb{R}$ at $t=0$. At a given time $t>0$, the position of a random Brownian walker is then $x=\alpha+v t+\sigma \sqrt{t} g$ where $g$ is a Gaussian random variable with zero mean and unit variance. Let us now introduce a second random variable $p>0$ which is independent of $g$ and follows a generic distribution $\mathcal{P}_{p}$. We use $p$ to pick random times at which we sample the positions of the Brownian particles. This leads us to consider the random variable $x=$ $\alpha+\beta p+\sigma \sqrt{p} g$ which is a non-linear combination of $g$ and p. Its $\mathrm{PDF}$,

$\mathcal{P}_{x}(x)=\mathcal{N}_{x}\left(x ; \alpha+\beta p, \sigma^{2} p\right) \circ \mathcal{P}_{p}(p)$,

is called a normal variance-mean mixture (Barndorff-Nielsen et al. 1982). A theorem shows that if $p$ is unimodal then so is $x$ (Yu 2011). 


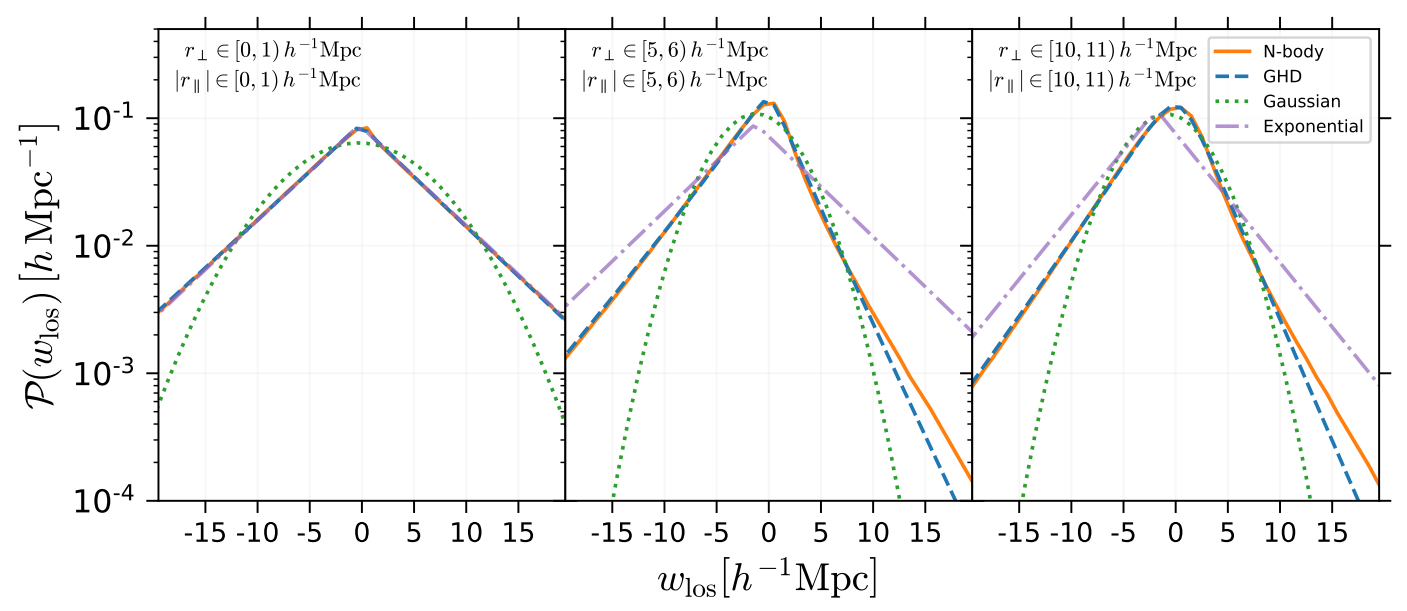

Figure 10. The los pairwise velocity distribution for the DM particles in the W0 simulation at $z=0$ (solid) is compared with the best-fitting GH (dashed), exponential (dot-dashed) and Gaussian (dotted) approximations for three different pair-separation distances in real space.
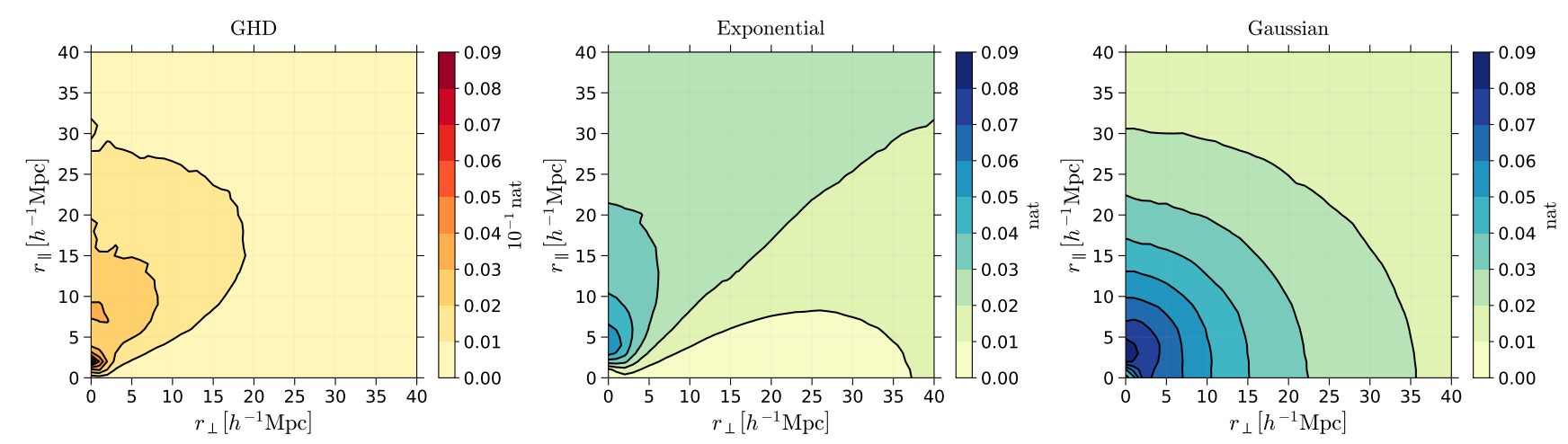

Figure 11. KL divergence for the GHD (left), exponential (middle) and Gaussian (right) best-fitting functions with respect to the los pairwise velocity distribution measured in the simulation. The reported values are expressed in natural units of information. Note that the color bar in the left panel is compressed by a factor of 10 compared with the middle and right panel.

\subsubsection{The generalised hyperbolic distribution}

A Gaussian PDF plotted on a semi-logarithmic graph describes a parabola. Although the frequency distribution of many empirical phenomena shows this property, there exist cases in which an hyperbola provides a much better description than a parabola due to the presence of exponential tails. A classic example is the log-size distribution of sand grains in natural aeolian deposits (Bagnold 1941) and many others arise particularly in finance. The name 'hyperbolic distributions' has been coined to designate this class of probability densities. The very first analytical example of such a PDF was derived in physics by calculating the distribution of particle velocities in an ideal relativistic gas (Jüttner 1911).

The GHD is a larger family of PDFs that includes the hyperbolic distributions as a particular case. It was introduced by Barndorff-Nielsen (1977) in order to model the log-size distribution of sand grains and is defined as a normal variance-mean mixture in which $\sigma=1$ and $\mathcal{P}_{p}(p)=$
$\mathcal{G}_{p}\left(p ; \lambda, \delta^{2}, \alpha^{2}-\beta^{2}\right)$. Its PDF takes the form (Prause 1999)

$$
\mathcal{H}_{x}(x ; \alpha, \beta, \delta, \lambda, \mu)=\mathcal{N}_{x}(x ; \mu+\beta p, p) \circ \mathcal{G}_{p}\left(p ; \lambda, \delta^{2}, \sqrt{\alpha^{2}-\beta^{2}}\right)=
$$$$
C\left[\delta^{2}+(x-\mu)^{2}\right]^{\frac{\lambda-1 / 2}{2}} e^{\beta(x-\mu)} K_{\lambda-\frac{1}{2}}\left(\alpha \sqrt{\delta^{2}+(x-\mu)^{2}}\right),
$$

with normalisation constant

$C=\frac{\left(\alpha^{2}-\beta^{2}\right)^{\frac{\lambda}{2}}}{\sqrt{2 \pi} \alpha^{\lambda-1 / 2} \delta^{\lambda} K_{\lambda}\left[\delta \sqrt{\alpha^{2}-\beta^{2}}\right]}$.

The domain of variation of the parameters is

$\delta \geq 0, \quad|\beta|<\alpha, \quad$ if $\lambda>0$,

$\delta>0, \quad|\beta|<\alpha, \quad$ if $\lambda=0$,

$\delta>0,|\beta| \leq \alpha, \quad$ if $\lambda<0$.

It is not easy to isolate the impact of each of them and several alternative parameterizations of the GHD have been introduced to alleviate this problem. Broadly speaking, $\lambda$ defines various subclasses and influences the tails, $\alpha$ modifies the shape (i.e. variance and kurtosis), $\beta$ the skewness, $\delta$ the 


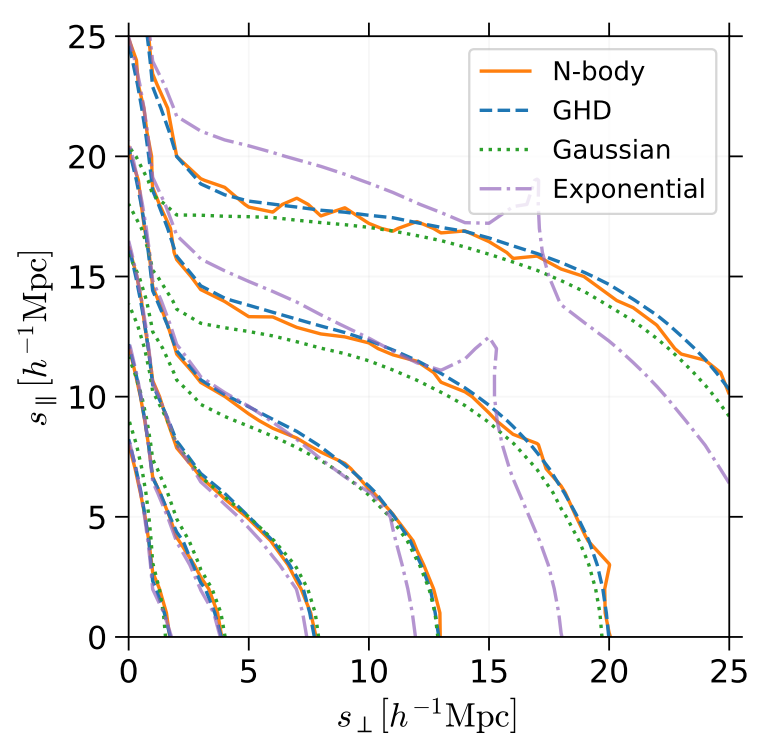

Figure 12. The redshift-space correlation function $\xi_{\mathrm{s}}$ for the particles in our $N$-body simulations (solid) is compared with the outcome of the streaming model obtained by fitting either a GHD (dashed), a Gaussian distribution (dotted) or an exponential (dash-dotted) to $\mathcal{P}_{w_{\text {los }}}\left(w_{\text {los }} \mid \mathbf{r}\right)$.

scale and $\mu$ shifts the mean value. A convenient property of the GHD is that it reduces to several named distributions in the appropriate limit. For example, it gives the hyperbolic distribution for $\lambda=1$ and becomes a Gaussian distribution with variance $\delta / \alpha$ when both $\alpha$ and $\delta$ tend to infinity (Hammerstein 2011).

The GHD shows semi-heavy tails (Barndorff-Nielsen \& Blaesild 1981),

$\mathcal{H}_{x} \sim|x|^{\lambda-1} \exp (-\alpha|x|+\beta x) \quad$ as $\quad x \rightarrow \pm \infty$,

and all its moments exist. The moment generating function is

$M(x)=e^{\mu x}\left[\frac{\alpha^{2}-\beta^{2}}{\alpha^{2}-(\beta+x)^{2}}\right]^{\lambda / 2} \frac{K_{\lambda}\left(\delta \sqrt{\alpha^{2}-(\beta+x)^{2}}\right)}{K_{\lambda}\left(\delta \sqrt{\alpha^{2}-\beta^{2}}\right)}$,

with $|\beta+x|<\alpha$ which follows from equation (49). Explicit expressions for the first four moments and cumulants are given in Barndorff-Nielsen \& Blaesild (1981).

\subsection{Application to pairwise velocities}

\subsubsection{DM particles}

In Fig. 10, we show that the GHD (dashed line) provides a very good fit to the histogram of $w_{\text {los }}$ extracted from the W0 simulation (solid line). The optimal values for the parameters have been determined assuming (symmetrised) Poisson errors (Gehrels 1986) and using least-squares fitting with a Markov Chain Monte Carlo sampler (we have checked for a few separations that this method gives consistent results with a maximum-likelihood analysis which is time consuming given the huge number of particle pairs). Our results show that the GHD accurately describes $\mathcal{P}_{\boldsymbol{w}_{\text {los }}}$ around the mode and in the negative tail while it slightly underestimates the PDF in the positive tail. The improvement with respect to Gaussian fits (dotted lines) is dramatic as the normal distribution cannot match the exponential tails seen in the simulation. For very small spatial separations, the exponential distribution given in equation (30) also provides a very good fit (dot-dashed lines). However, the agreement with the simulation data rapidly decreases with increasing $r$ as the model has the wrong shape around the mode of the distribution. A more quantitative analysis is performed in Fig. 11 where we compare the information loss associated with the GHD, exponential and Gaussian approximations. Shown is the Kullback-Leibler (KL) divergence between the actual PDF measured in the simulations and the three approximations as a function of the real-space separation of the pairs. This quantity provides a measure of goodness of fit. For the range of separation vectors shown in Fig. 11, the information loss associated with the Gaussian approximation is always at least one order of magnitude larger than for the GHD. This property persists also on larger scales. Similar conclusions can be drawn comparing the exponential and the GHD approximations, although, in this case, the fits are of similar quality at very small separations $\left(r_{\|}<1 h^{-1} \mathrm{Mpc}\right.$ and $\left.r_{\perp}<15 h^{-1} \mathrm{Mpc}\right)$. Note that the GHD compression is nearly lossless at all scales.

Finally, in Fig. 12 we show the redshift-space correlation function obtained with the streaming model by inserting the best-fitting GHD in equation (22) together with the realspace correlation function extracted from the simulation. Our results (dashed lines) provide an excellent description of the redshift-space correlation measured in the simulation (solid lines). For $s>5 h^{-1} \mathrm{Mpc}$, deviations are comparable with the Poisson error for $\xi_{\text {s }}$ which is always between one and a few per cent. On smaller scales, where the Poisson error becomes substantially sub per cent, one starts noticing statistically significant deviations at a few per cent level (not visible in the plot). For comparison, we also show the results obtained using the Gaussian and exponential fits for $\mathcal{P}_{w_{\text {los }}}$. The Gaussian model (dotted lines) shows large systematic deviations on small scales and matches the simulations (at the level of the Poisson errors) only for $s_{\|}>20 h^{-1} \mathrm{Mpc}$ and $s_{\perp}>5 h^{-1} \mathrm{Mpc}$. The exponential model ${ }^{6}$ (dot-dashed lines), on the other hand, is accurate only at small $s_{\perp}$.

The high fidelity of the GHD fit comes at the price of using five free parameters. Their marked scale dependence (see Fig. 13) represents a severe limitation for future practical applications that aim to interpret observational data. We note, however, that the best-fitting parameters at different $\mathbf{r}$ tightly cluster along a flattened sequence in five-dimensional space. By applying a principal component analysis to the standardised variables, we find that the first three components account for 99.8 per cent of the variance. We thus fit again the distribution of $w_{\text {los }}$ in the simulation using only three free parameters that denote the position of $\alpha, \beta, \delta, \lambda$ and $\mu$ within the space spanned by the first three principal components. In Fig. 14, we show the quality of the best-fitting

6 The features that are noticeable in the contour map of $\xi_{\mathrm{s}}$ at $s_{\|} \simeq s_{\perp}$ are caused by discontinuities in $\bar{w}_{\|}$and $\sigma_{12}$ as a function of $r_{\|}$and $r_{\perp}$. In fact, the posterior probability density of these parameters is bimodal for a range of real-space separations and we selected the peak with the highest integrated probability to draw the dot-dashed lines in Fig. 12. 

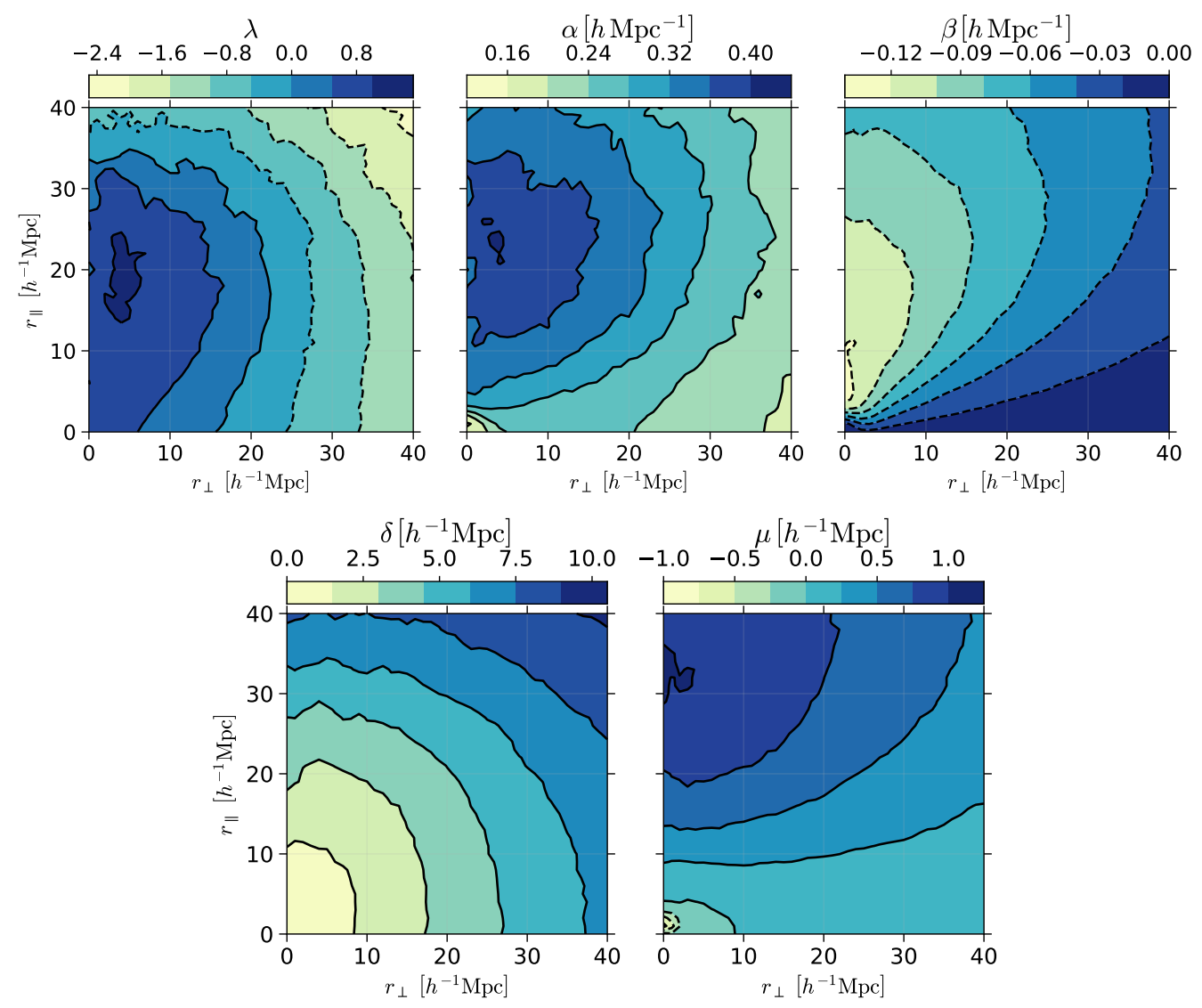

Figure 13. Best-fitting parameters of the GHD as a function of $r_{\|}$and $r_{\perp}$.

functions as well as the corresponding $\xi_{\mathrm{s}}$ obtained by inserting them into equation (22). The three-parameter GHD still outperforms the Gaussian approximation at all scales. This requirement can be relaxed at larger scales where $\mathcal{P}_{w_{\text {los }}}$ assumes a simpler shape. Fig. 15 shows that a two-parameter GHD obtained through PCA for larger scales provides an excellent fit that better describes the tails of the distribution with respect to the Gaussian approximation at all spatial separations. Note that even the integrand of the streaming equation is impeccably reproduced in this case.

\subsubsection{DM haloes}

DM haloes are biased tracers of the matter distributions and, as discussed in Section 5.2.2, are also characterized by a different pairwise-velocity PDF which presents less prominent tails than for the matter. In fact, haloes are not subject to the Finger-of-God effect and have a substantially smaller pairwise velocity dispersion, especially at small separations. In Fig. 16, we show that the GHD provides an excellent fit to the halo pairwise velocity distribution and, also in this case, outperforms the Gaussian approximation.

\subsection{Discussion}

\subsubsection{Comparison with previous work}

The GHD is a mixture of Gaussians analogous to that introduced in Bianchi et al. (2015), although with a very different mixing distribution. It is thus interesting to highlight similarities and differences between the two approaches. In both cases, skewness is generated by the correlation between the mean and the variance of the constituent Gaussian distributions. However, the two models achieve this differently. In Bianchi et al. (2015), $\mu$ and $\sigma$ are Gaussian random variables and their correlation is a free parameter. The GHD instead originates from the deterministic relation $\mu=\alpha+\beta \sigma^{2}$. Additional skewness appears in the GHD because the mixing distribution $\mathcal{G}$ is itself skewed. This illustrates why the GHD can accommodate the large skewness measured in $N$-body simulations at small $r$ while the model by Bianchi et al. (2015) cannot (see Bianchi et al. 2016).

Another difference between the two approaches lies in the support of the mixing distribution. Although the rms value $\sigma$ is by definition non-negative, Bianchi et al. (2015) use a mixing function with support on $\mathbb{R}^{2}$ for $\mu$ and $\sigma$. The mixing integral therefore extends over unphysical regions where $\sigma<0$. A convenient fix is to truncate the Gaussian mixing distribution at $\sigma=0$ (and renormalise it) as proposed in Bianchi et al. (2016). On the other hand, the GHD is based on a non-Gaussian mixing distribution with positive support for $\sigma^{2}$. In brief, while Bianchi et al. (2016) use a mixture of (slightly) non-Gaussian distributions with (truncated) Gaussian mixing, we use a mixture of normals with a strongly non-Gaussian mixing distribution.

Our approach offers multiple benefits: i) the GHD has long been studied and its mathematical properties are well 

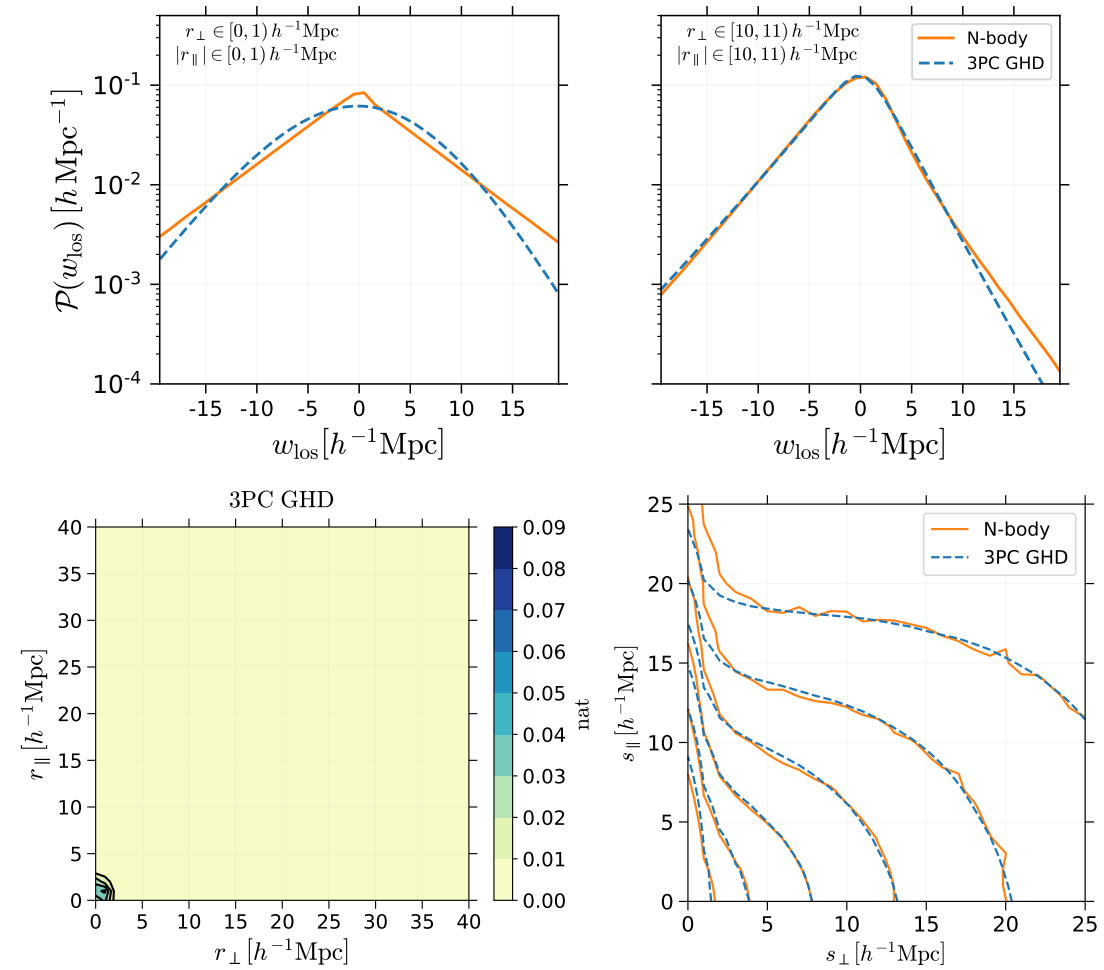

Figure 14. As in Figs. 10, 11 and 12 but for a GHD with only 3 degrees of freedom that identify the position of the model parameters within the volume spanned by the first 3 principal components.
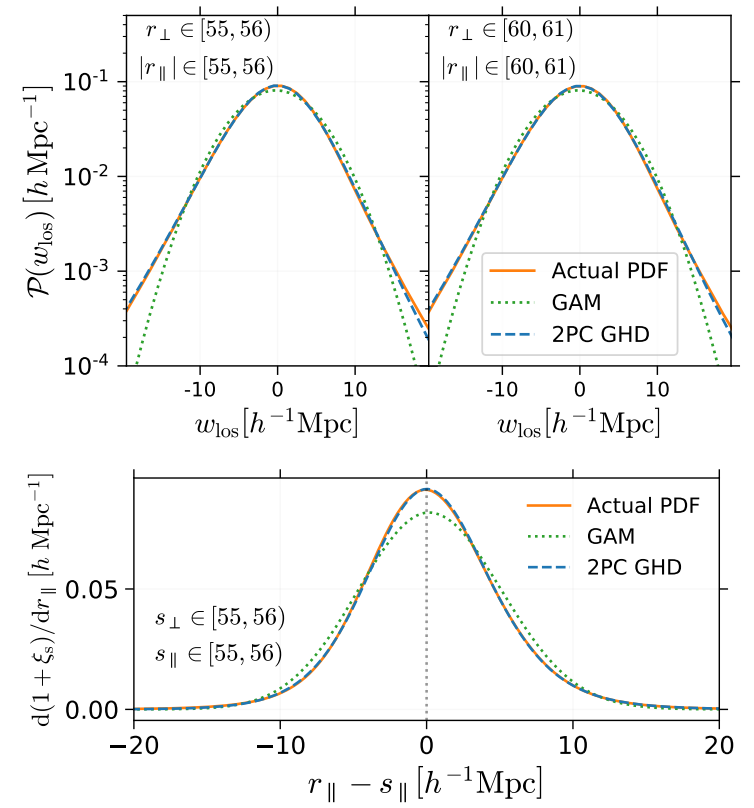

Figure 15. A simplified GHD model for $\mathcal{P}_{w_{\text {los }}}$ in which the number of free parameters is reduced to 2 through PCA (2PC GHD, dashed) provides an excellent description of the $N$-body data (solid) on large scales and vastly outperforms the Gaussian approximation (dotted). In the top panel, we directly compare data and best-fit models for the pairwise-velocity PDF (as in Fig. 10 but for larger pair separations given in units of $h^{-1} \mathrm{Mpc}$ ). In the bottom panel, we show the corresponding integrand of the streaming equation, as in Fig. 4. known; ii) it has an analytical expression with several different parameterisations; iii) its moment generating function is analytical and expressions are available for its first four cumulants; iv) it reduces to the Gaussian distribution in some limit; v) optimised techniques have been developed for estimating its parameters given a set of data; vi) packages in the most popular computer languages are available for its efficient evaluation and also for the estimation of its parameters.

\subsubsection{Cosmology dependence}

Current cosmic-microwave-background experiments and large-scale-structure studies have set tight constraints on the cosmological parameters of the $\Lambda \mathrm{CDM}$ model. One open question is whether the pairwise velocity PDF changes significantly when the underlying cosmological model is varied within the currently allowed region of parameter space. We investigate this issue in Fig. 17 where we compare the pairwise velocity PDFs at $z=0$ for the DM particles extracted from the 6 simulations introduced in Section 2. Switching from the WMAP to the Planck cosmology or considering non-Gaussian initial fluctuations (even at a level that violates current observational limits) only introduces minimal changes in $\mathcal{P}_{w_{\text {los }}}$ at all separation vectors. Minute differences are noticeable only in the high-velocity tails. This is good news as it means that it should be possible to parameterize the scale-dependence of the PDF in a cosmologyindependent way so that to facilitate practical applications of the GHD model. 


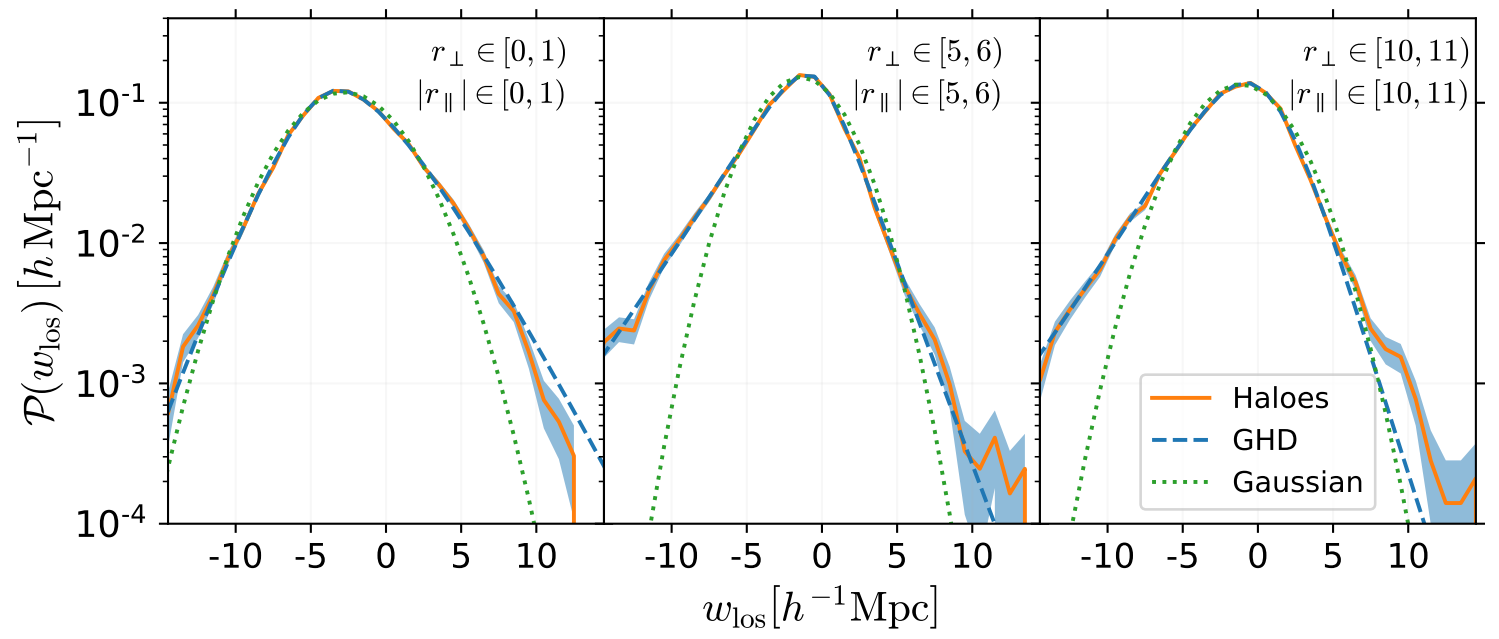

Figure 16. The los pairwise velocity distribution for the DM haloes identified in the W0 simulation at $z=0$ (solid line with shaded error) is compared with the best-fitting GH (dashed) and Gaussian (dotted) approximations for diffferent real-space pair separations expressed in $h^{-1} \mathrm{Mpc}$.

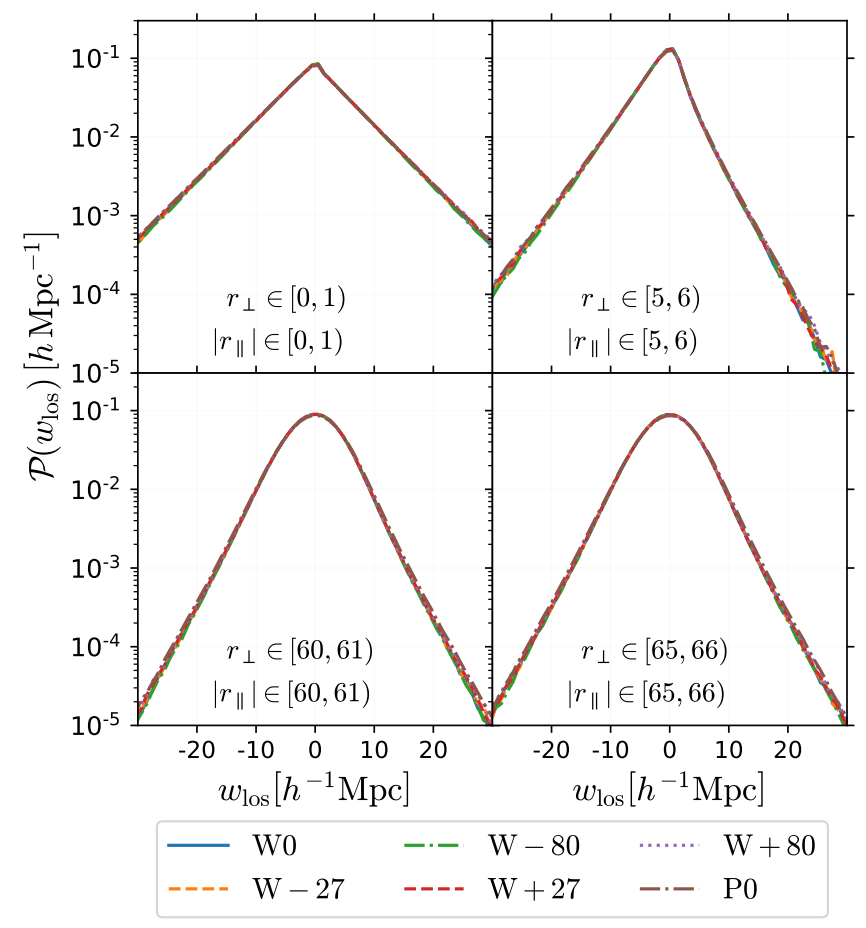

Figure 17. The los pairwise velocity distribution measured at $z=$ 0 for the DM particles in the six cosmological models introduced in Table 1. Spatial separations are given in units of $h^{-1} \mathrm{Mpc}$.

\subsubsection{Redshift evolution}

The pairwise-velocity PDF for the DM particles reflects the non-linear growth of the large-scale structure of the Universe and, therefore, evolves with time. This is shown in Fig. 18, where we use the W0 simulation to plot $\mathcal{P}_{w_{\text {los }}}$ for various real-space separations at four different epochs. At $z=2$, the PDF already exhibits non-Gaussian features including asymmetry around the mode. However, the PDF is much more strongly peaked and presents less prominent exponen-

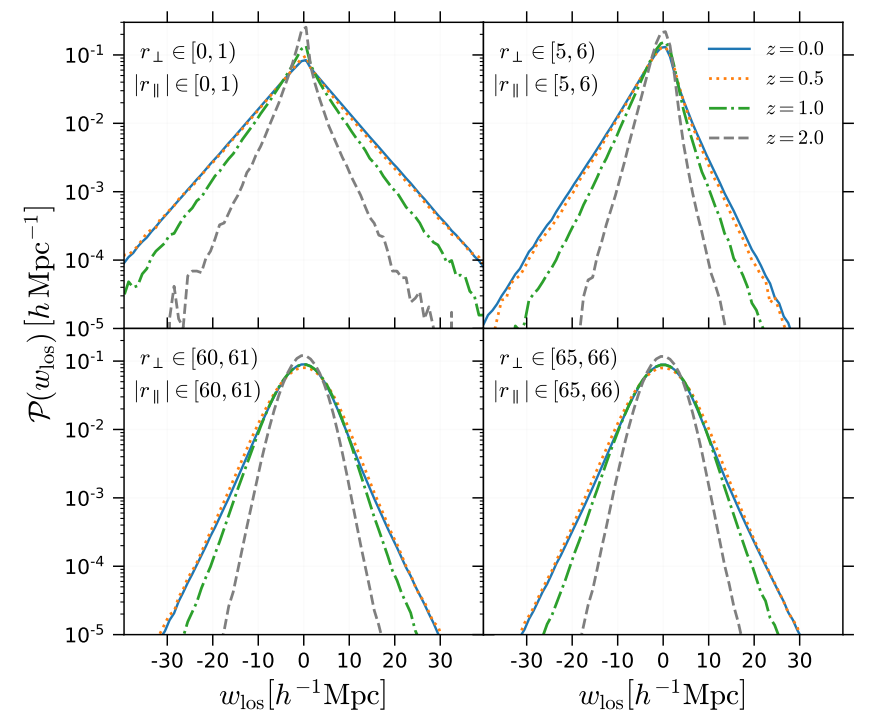

Figure 18. Redshift evolution of the los pairwise velocity distribution for the DM particles of the W0 simulation. Real-space separations are given in units of $h^{-1} \mathrm{Mpc}$.

tial tails than at $z=0$. In fact, only rare massive haloes are resolved at early times and the velocity PDF is dominated by field-field pairs. As time goes by, more and more haloes above the mass-resolution limit form and the velocity PDF develops fatter tails. It is worth stressing that almost no evolution is noticeable between $z=0.5$ and $z=0$. This is a consequence of the fact that the energy budget of the universe becomes dominated by the cosmological constant and further development of structure is inhibited. Combined with the results of Section 6.3.2, this finding implies that, for $z \lesssim 0.5$, it should be possible to define a cosmologyand redshift-independent parameterization of the pairwisevelocity PDF, at least for the DM particles. 


\subsubsection{DM vs galaxies}

The ultimate application of the streaming model is the interpretation of the clustering signal extracted for galaxy redshift surveys. However, our study (like many others before) focusses on the analysis of simulated DM particles and haloes. The advantage of using the simulation particles is that they offer a huge statistical sample. On the other hand, the PDF of their pairwise velocities shows stronger exponential tails with respect to haloes due to the broadening generated by virial motions and spikier peaks due to diffuse matter (see Fig. 8). In a sense, a galaxy sample is expected to show intermediate properties between halo and particle datasets. A pure sample of central galaxies will closely look like a halo sample while adding more and more satellites will progressively drive the PDF towards the results for simulation particles. It is long known that galaxy clustering in redshift space is well described by the streaming model assuming an exponential $\mathcal{P}_{w_{\text {los }}}$ on very small scales and a Gaussian one on very large scales. Since the GHD is of very general form and reduces to these limits for particular combinations of its parameters, we are confident that our analysis can be straightforwardly generalised to galaxies. We will investigate this issue in our future work.

\section{SUMMARY}

The galaxy 2-point correlation function in redshift space depends on the orientation of the pair-separation vector with respect to the los. This anisotropy encodes information about the velocities arising from gravitational instability. On large scales, where linear perturbation theory applies, RSD allow for a measurement of the growth rate of structure. Combining estimates at different redshifts help differentiate dark energy models based on General Relativity from modified gravity as the cause of the accelerating Universe.

Modifications to the theory of gravity generally introduce extra degrees of freedom whose effect (the so-called fifth force) must be suppressed by some screening mechanism on scales where General Relativity is well tested. Such constraint implies that characteristic signatures will be imprinted on intermediate cosmological scales. In fact, the screening mechanism is expected to affect the non-linear clustering and the velocities of tracers of the large-scale structure. Testing these predictions provides a strong motivation to extend the analysis of galaxy clustering to smaller scales than currently done in cosmological studies.

Realising this program in practice requires, however, a number of tools. Among them, robust theoretical predictions for the modified theories of gravity together with an accurate (and, possibly, non-perturbative) description of RSD. In this paper, we focussed on the latter issue. In particular, we discussed the classical streaming model for RSD and how its implementation can be improved to get accurate predictions at non-linear scales. Our main results can be summarised as follows.

In Section 4, starting from the one- and two-particle phase-space densities, we derived the governing equations of the model. For ordered pairs, we obtained equation (14) which coincides with the standard equation discussed in the literature. Our result is exact and holds true also in the case of multi streaming thanks to our particle-based approach. The correct solution for unordered pairs has been given in equation (22). The modifications with respect to equation (14) account for the pairs that reverse their los ordering between real and redshift space. These swaps occur more frequently for pairs with small spatial separations.

After briefly reviewing the history of the streaming model and of its applications, we investigated the limitations of using the Gaussian ansatz for the pairwise-velocity PDF. In agreement with previous studies, we showed that this approximation fails to reproduce the outcome of $N$-body simulations for redshift-space separations $s \lesssim 20 h^{-1} \mathrm{Mpc}$ while it achieves percent level accuracy on $\xi_{\mathrm{s}}$ on substantially larger scales. Our analysis revealed, however, that a Gaussian PDF never manages to reproduce the integrand of the streaming equation to the same level of accuracy. The success of the GSM on large scales, therefore, originates from fortuitous cancellations between the contributions of the peak and the wings in the integrand of the streaming equation (see Fig. 4).

In Section 5, we used a high-resolution $N$-body simulation to investigate how the PDF of the los component of the pairwise-velocity, $w_{\|}$, depends on the pair separation vector for both DM particles and haloes. The first four cumulants of the PDF show a complex pattern (Fig. 5) that can be understood in terms of a few isotropic components and the angle that the pair separation forms with the los. We derived a general relation between the cumulants of $w_{\|}$ and those of the radial and transverse pairwise velocities, equation (37). We then studied the scale-dependence of the isotropic components in the simulation (Fig. 6) and measured the pairwise-velocity bias of the DM haloes (Fig. 7). Additionally, we dissected the pairwise-velocity PDF for the DM and showed that the tails are generated by particles in massive haloes while the region around the mode is dominated by field particles (Figs. 8 and 9).

Finally, in Section 6, we proposed an analytical fitting function for the pairwise-velocity distribution and demonstrated that it provides an excellent description of numerical data. We first introduced the mathematical background of mixtures and then described the properties of the GHD, a unimodal PDF with exponential tails. Comparing with $N$-body simulations, we showed that the GHD is able to approximate the PDF of the los pairwise velocity at all scales (for both DM particles and haloes) with minimal information loss compared to the common exponential and Gaussian fits (Figs. 10, 11, and 12). The main drawback to using the GHD in future practical applications is that it depends on 5 tunable parameters. In fact, fixing their values to best fit the $N$-body data gives rise to non-trivial scale dependencies (Fig. 13). However, the best-fitting values are strongly correlated and tend to populate a lower-dimensional sequence in 5-dimensional parameter space. Using principal-component analysis to exploit the correlations, we managed to reduce the complexity of the model while still providing a remarkable fit to $\xi_{\mathrm{s}}$. We found that 2 parameters are enough on large scales $\left(s>70 h^{-1} \mathrm{Mpc}\right)$ while at least 3 are needed on smaller scales. In this case, even the integrand of the streaming equation is accurately reproduced by the model (Fig. 15).

Intriguingly, we found that the pairwise-velocity PDF for the DM at $z=0$ shows only minimal changes when the underlying cosmological parameters are varied within the 
current constraints for the $\Lambda \mathrm{CDM}$ model (Fig. 17). Moreover, it does not show any noticeable time evolution for redshifts $z \lesssim 0.5$ (Fig. 18). All this suggests that, for lowredshift tracers, it should be possible to find a cosmologyand redshift-independent parameterization for the PDF of their pairwise velocities as a function of the separation vector. This would greatly simplify the implementation of the GHD model for studying anisotropic galaxy clustering. We will investigate the applicability of these findings to forthcoming data in our future work.

\section{ACKNOWLEDGEMENTS}

We acknowledge partial financial support from the Deutsche Forschungsgemeinschaft through the Bonn-Cologne Graduate School for Physics and Astronomy and the Transregio 33 'The Dark Universe'. We are thankful to the community for developing and maintaining open-source software packages extensively used in our work, namely Cython (Behnel et al. 2011), emcee (Foreman-Mackey et al. 2013), Matplotlib (Hunter 2007), Numpy (van der Walt et al. 2011), Scikitlearn (Pedregosa et al. 2011) and Scipy (Jones et al. 2001).

\section{REFERENCES}

Abramowitz M., Stegun I. A., 1972, Handbook of Mathematical Functions

Agrawal A., Makiya R., Chiang C.-T., Jeong D., Saito S., Komatsu E., 2017, preprint, (arXiv:1706.09195)

Alam S., Ho S., Vargas-Magaña M., Schneider D. P., 2015, MNRAS, 453, 1754

Arnalte-Mur P., Hellwing W. A., Norberg P., 2017, MNRAS, 467, 1569

Bagnold R., 1941, The Physics of Blown Sand and Desert Dunes. Methuen

Barndorff-Nielsen O., 1977, Proceedings of the Royal Society of London A: Mathematical, Physical and Engineering Sciences, 353, 401

Barndorff-Nielsen O., Blaesild P., 1981, Hyperbolic Distributions and Ramifications: Contributions to Theory and Application. Springer Netherlands, Dordrecht, pp 19-44, doi:10.1007/978-94-009-8549-0_2, https://doi.org/10.1007/ 978-94-009-8549-0_2

Barndorff-Nielsen O., Kent J., Sorensen M., 1982, Int. Stat. Rev., 50,145

Barreira A., Sánchez A. G., Schmidt F., 2016, Phys. Rev. D, 94, 084022

Bean A. J., Ellis R. S., Shanks T., Efstathiou G., Peterson B. A., 1983, MNRAS, 205, 605

Behnel S., Bradshaw R., Citro C., Dalcin L., Seljebotn D. S., Smith K., 2011, Computing in Science \& Engineering, 13, 31

Behroozi P. S., Wechsler R. H., Wu H.-Y., 2013, ApJ, 762, 109

Beutler F., et al., 2012, MNRAS, 423, 3430

Bianchi D., Chiesa M., Guzzo L., 2015, MNRAS, 446, 75

Bianchi D., Percival W. J., Bel J., 2016, MNRAS, 463, 3783

Bond J. R., Cole S., Efstathiou G., Kaiser N., 1991, ApJ, 379, 440

Borzyszkowski M., Bertacca D., Porciani C., 2017, MNRAS, 471, 3899

Carlberg R. G., 1994, ApJ, 433, 468

Carlberg R. G., Couchman H. M. P., 1989, ApJ, 340, 47

Carlson J., Reid B., White M., 2013, MNRAS, 429, 1674

Cen R., Ostriker J. P., 1992, ApJ, 399, L113
Chhikara R. S., Folks J. L., 1989, The Inverse Gaussian Distribution: Theory, Methodology, and Applications. Marcel Dekker, Inc., New York, NY, USA

Chuang C.-H., Wang Y., 2013, MNRAS, 431, 2634

Chuang C.-H., et al., 2017, MNRAS, 471, 2370

Colín P., Klypin A. A., Kravtsov A. V., 2000, ApJ, 539, 561

Couchman H. M. P., Carlberg R. G., 1992, ApJ, 389, 453

Davis M., Peebles P. J. E., 1977, ApJS, 34, 425

Davis M., Peebles P. J. E., 1983, ApJ, 267, 465

Efstathiou G., Frenk C. S., White S. D. M., Davis M., 1988, MNRAS, 235, 715

Evrard A. E., Summers F. J., Davis M., 1994, ApJ, 422, 11

Fisher K. B., 1995, ApJ, 448, 494

Fisher K. B., Davis M., Strauss M. A., Yahil A., Huchra J. P., 1994, MNRAS, 267, 927

Foreman-Mackey D., Hogg D. W., Lang D., Goodman J., 2013, PASP, 125, 306

Gehrels N., 1986, ApJ, 303, 336

Gelb J. M., Bertschinger E., 1994, ApJ, 436, 491

Giannantonio T., Porciani C., Carron J., Amara A., Pillepich A., 2012, MNRAS, 422, 2854

Guzzo L., Strauss M. A., Fisher K. B., Giovanelli R., Haynes M. P., 1997, ApJ, 489, 37

Guzzo L., et al., 2008, Nature, 451, 541

Hale-Sutton D., Fong R., Metcalfe N., Shanks T., 1989, MNRAS, 237,569

Hamilton A. J. S., 1998, in Hamilton D., ed., Astrophysics and Space Science Library Vol. 231, The Evolving Universe. p. 185 (arXiv:astro-ph/9708102), doi:10.1007/978-94-0114960-0_17

Hammerstein E. A. v., 2011, Generalized hyperbolic distributions: theory and applications to CDO pricing, https://freidok. uni-freiburg.de/data/7974

Hawkins E., et al., 2003, MNRAS, 346, 78

Hellwing W. A., Barreira A., Frenk C. S., Li B., Cole S., 2014, Physical Review Letters, 112, 221102

Hunter J. D., 2007, Computing in Science \& Engineering, 9, 90

Jackson J. C., 1972, Monthly Notices of the Royal Astronomical Society, 156, 1P

Jennings E., Baugh C. M., Li B., Zhao G.-B., Koyama K., 2012, MNRAS, 425, 2128

Jing Y. P., Börner G., Suto Y., 2002, ApJ, 564, 15

Jones E., Oliphant T., Peterson P., et al., 2001, SciPy: Open source scientific tools for Python, http://www.scipy.org/

Jørgensen B., 1982, Statistical Properties of the Generalized. Lecture Notes in Statistics Vol. 9, Springer New York, doi:10.1007/978-1-4612-5698-4, https://link. springer. com/book/10.1007\%2F978-1-4612-5698-4

Juszkiewicz R., Fisher K. B., Szapudi I., 1998, ApJ, 504, L1

Jüttner F., 1911, Annalen der Physik, 339, 856

Kaiser N., 1987, MNRAS, 227, 1

Kepner J. V., Summers F. J., Strauss M. A., 1997, New Astron., 2,165

Komatsu E., et al., 2009, ApJS, 180, 330

Kopp M., Uhlemann C., Achitouv I., 2016, Phys. Rev. D, 94, 123522

Kwan J., Lewis G. F., Linder E. V., 2012, ApJ, 748, 78

Li C., Jing Y. P., Kauffmann G., Börner G., White S. D. M., Cheng F. Z., 2006, MNRAS, 368, 37

Li C., Jing Y. P., Kauffmann G., Börner G., Kang X., Wang L., 2007, MNRAS, 376, 984

Loveday J., Efstathiou G., Maddox S. J., Peterson B. A., 1996, ApJ, 468, 1

Marulli F., Baldi M., Moscardini L., 2012, MNRAS, 420, 2377

Marzke R. O., Geller M. J., da Costa L. N., Huchra J. P., 1995, AJ, 110, 477

Matsubara T., 2008, Phys. Rev. D, 78, 083519

Mo H. J., Jing Y. P., Borner G., 1993, MNRAS, 264, 825 
Park C., Vogeley M. S., Geller M. J., Huchra J. P., 1994, ApJ, 431, 569

Peacock J. A., Dodds S. J., 1994, MNRAS, 267, 1020

Peacock J. A., West M. J., 1992, MNRAS, 259, 494

Peacock J. A., et al., 2001, Nature, 410, 169

Pedregosa F., et al., 2011, Journal of Machine Learning Research

Peebles P. J. E., 1976, Ap\&SS, 45, 3

Peebles P. J. E., 1979, AJ, 84, 730

Peebles P. J. E., 1980, The large-scale structure of the universe

Pillepich A., Porciani C., Hahn O., 2010, MNRAS, 402, 191

Planck Collaboration et al., 2016, A\&A, 594, A1

Prause K., 1999, The Generalized Hyperbolic Model: Estimation, Financial Derivatives, and Risk Measures, https://freidok. uni-freiburg.de/data/15

Reid B. A., White M., 2011, MNRAS, 417, 1913

Reid B. A., et al., 2012, MNRAS, 426, 2719

Rostoker N., Rosenbluth M. N., 1960, The Physics of Fluids, 3, 1

Sabiu C. G., Mota D. F., Llinares C., Park C., 2016, A\&A, 592, A38

Samushia L., et al., 2014, MNRAS, 439, 3504

Sargent W. L. W., Turner E. L., 1977, ApJ, 212, L3

Satpathy S., et al., 2017, MNRAS, 469, 1369

Schrödinger E., 1915, Physikalische Zeitschrift, 16, 289

Scoccimarro R., 2004, Phys. Rev. D, 70, 083007

Seljak U., McDonald P., 2011, J. Cosmology Astropart. Phys., 11, 039

Senatore L., Zaldarriaga M., 2014, preprint, (arXiv:1409.1225)

Seshadri V., 1999, The inverse Gaussian distribution: statistical theory and applications. Lecture notes in statistics, Springer, https://link.springer.com/book/10.1007\% 2F978-1-4612-1456-4

Shepherd C. W., Carlberg R. G., Yee H. K. C., Ellingson E., 1997, ApJ, 479, 82

Sheth R. K., 1996, MNRAS, 279, 1310

Sheth R. K., Diaferio A., 2001, MNRAS, 322, 901

Sichel H. S., 1974, Journal of the Royal Statistical Society, Series A, pp 25-34

Springel V., 2005, MNRAS, 364, 1105

Springel V., Yoshida N., White S. D. M., 2001, New Astron., 6, 79

Summers F. J., Davis M., Evrard A. E., 1995, ApJ, 454, 1

Taruya A., Nishimichi T., Saito S., Hiramatsu T., 2009, Phys. Rev. D, 80, 123503

Taruya A., Nishimichi T., Bernardeau F., 2013, Phys. Rev. D, 87, 083509

Taruya A., Nishimichi T., Bernardeau F., Hiramatsu T., Koyama K., 2014, Phys. Rev. D, 90, 123515

Tinker J. L., 2007, MNRAS, 374, 477

Tinker J. L., Weinberg D. H., Zheng Z., 2006, MNRAS, 368, 85

Tweedie M. C. K., 1956, Virginia Journal Science, 7, 160

Uhlemann C., Kopp M., Haugg T., 2015, Phys. Rev. D, 92, 063004

Vallois P., 1991, Bull. Sc. Math., 115

Vlah Z., Castorina E., White M., 2016, J. Cosmology Astropart. Phys., 12, 007

Wang L., Reid B., White M., 2014, MNRAS, 437, 588

White M., 2014, MNRAS, 439, 3630

White M., Song Y.-S., Percival W. J., 2009, MNRAS, 397, 1348

Xu L., 2015, Phys. Rev. D, 91, 063008

Yu Y., 2011, preprint, (arXiv:1106.2333)

Yvon J., 1935, Actualites Sci. et Ind., 203

Zehavi I., et al., 2002, ApJ, 571, 172

Zu Y., Weinberg D. H., 2013, MNRAS, 431, 3319

Zu Y., Weinberg D. H., Jennings E., Li B., Wyman M., 2014, MNRAS, 445, 1885

Zurek W. H., Quinn P. J., Salmon J. K., Warren M. S., 1994, ApJ, 431, 559

van der Walt S., Colbert S. C., Varoquaux G., 2011, Computing in Science \& Engineering, 13, 22

\section{APPENDIX A: REVERSED PAIRS}

In Fig. A1, we quantify the importance of pair reversals for the two-point correlation function in redshift space. After measuring $\xi(r)$ and $\mathcal{P}_{w_{\text {los }}}$ in our simulation, we apply equation (22) and obtain the solid contour levels for $\xi_{s}$ that nicely match those shown in the right-hand panel of Fig. 1. The dashed contours, instead, are computed integrating over the positive values of $r_{\|}$only, which corresponds to neglecting the reversed pairs. Not only this incomplete model underestimates $\xi_{\mathrm{s}}$ by a factor of two in the FoG regime $\left(s_{\perp}<2 h^{-1}\right.$ Mpc and $s_{\|} \gg s_{\perp}$ where reversed pairs are as many as the preserved ones), but also severely suppresses the redshiftspace correlation whenever $s_{\|} \ll s_{\perp}$. The reversed pairs thus give an important contribution on a vast range of redshiftspace separations that extend well beyond the typical size of DM haloes.

It is also interesting to explore what would happen if one would naively replace $\mathcal{P}_{w_{\|}}$with $\mathcal{P}_{w_{\text {los }}}$ in equation (14) or, equivalently, drop the sgn function in equation (22). The corresponding result for $\xi_{\mathrm{s}}$ is shown with dotted lines in Fig. A1 and it underestimates the actual correlation function on many scales although less severely than in the previous case. To further investigate the origin of the differences, in the top panel of Fig. A2, we consider a narrow range of redshift-space separations $\left(s_{\|} \in[5,6) h^{-1} \mathrm{Mpc}\right.$ and $s_{\perp} \in[2,3) h^{-1} \mathrm{Mpc}$ ) and plot the integrand of equation (22) as a function of $r_{\|}$(solid line). For comparison, we also indicate with a dashed line the integrand obtained neglecting the sgn function in the argument of $\mathcal{P}_{w_{\text {los }}}$ for $r_{\|}<0$. Although the reversed pairs are taken into account in the latter case, they are miscounted which leads to the underestimation of $\xi_{\mathrm{s}}$.

Note that the correct function $\mathrm{d}\left(1+\xi_{\mathrm{s}}\right) / \mathrm{d} r_{\|}$is discontinous at $r_{\|}=0$. This feature originates from the function $\mathcal{P}_{w_{\text {los }}}\left[\left(s_{\|}-r_{\|}\right) \operatorname{sgn}\left(r_{\|}\right) \mid \mathbf{r}\right]$ which is plotted in the middle panel of Fig. A2: the factor $\operatorname{sgn}\left(r_{\|}\right)$makes sure that the (continuous but asymmetric) velocity PDF is sampled at $w_{\text {los }}=s_{\|}-r_{\|}$ for $r_{\|} \rightarrow 0^{+}$and at $w_{\text {los }}=r_{\|}-s_{\|}$for $r_{\|} \rightarrow 0^{-}$. Also note that $\mathcal{P}_{w_{\text {los }}}$ presents a prominent peak around $r_{\|} \simeq s_{\|}$corresponding to pairs with relatively small relative los velocity. The enhancement of the region around $r_{\|} \sim 0$ in the full integrand is due to the term $1+\xi(r)$ which is plotted in the bottom panel of the figure. In simple words, real-space clustering boosts the contribution from close pairs with large pairwise los velocity.

It is worth mentioning that, for much larger redshiftspace separations (e.g. $s_{\|} \gg 20 h^{-1} \mathrm{Mpc}$ and $s_{\perp} \gg$ $20 h^{-1} \mathrm{Mpc}$ ), the peak around $r_{\|} \approx 0$ is suppressed and the impact of the reversed pairs becomes much less important. In this case, neglecting the sgn function in equation (22) or using $\mathcal{P}_{w_{\text {los }}}$ in equation (14) generates only small errors.

This paper has been typeset from a $\mathrm{T}_{\mathrm{E}} \mathrm{X} / \mathrm{LAT}_{\mathrm{E}} \mathrm{X}$ file prepared by the author. 


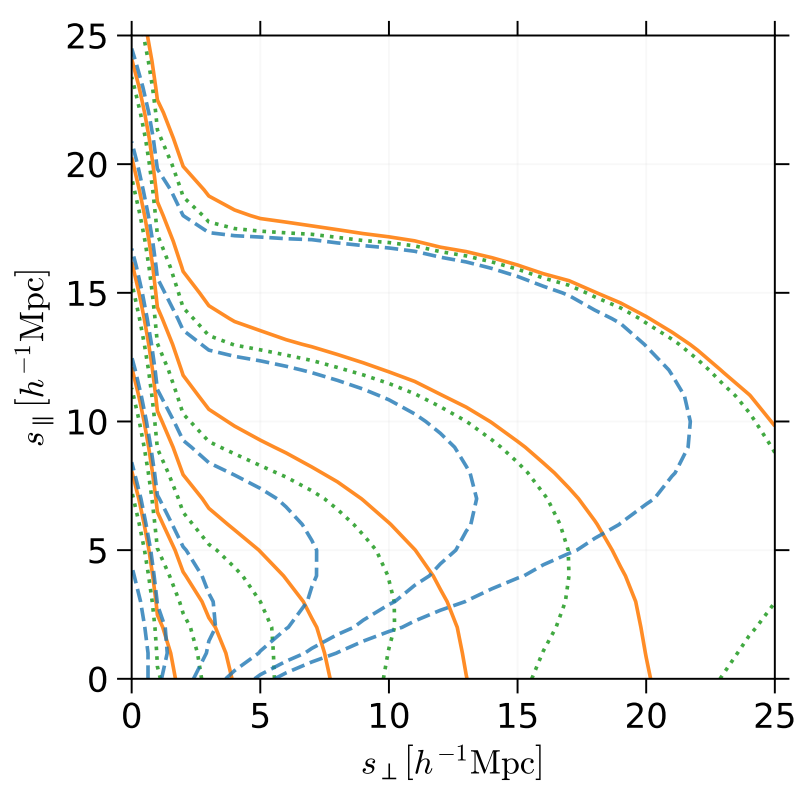

Figure A1. Impact of the reversed pairs on $\xi_{\mathrm{s}}$. The solid lines show the contour levels obtained using equation (22) which is exact for ordered pairs. In this case, the correlation function nicely matches the measurements presented in Fig. 1. This result is compared with two approximations that do not properly account for the reversed pairs. The dashed lines only considers the region with $r_{\|}>0$ in equation (22) and thus completely disregards the swapped pairs. The dotted lines, on the other hand, are obtained by replacing $\mathcal{P}_{w_{\|}}$with $\mathcal{P}_{w_{\text {los }}}$ in equation (14) which improperly weighs the reversed pairs.

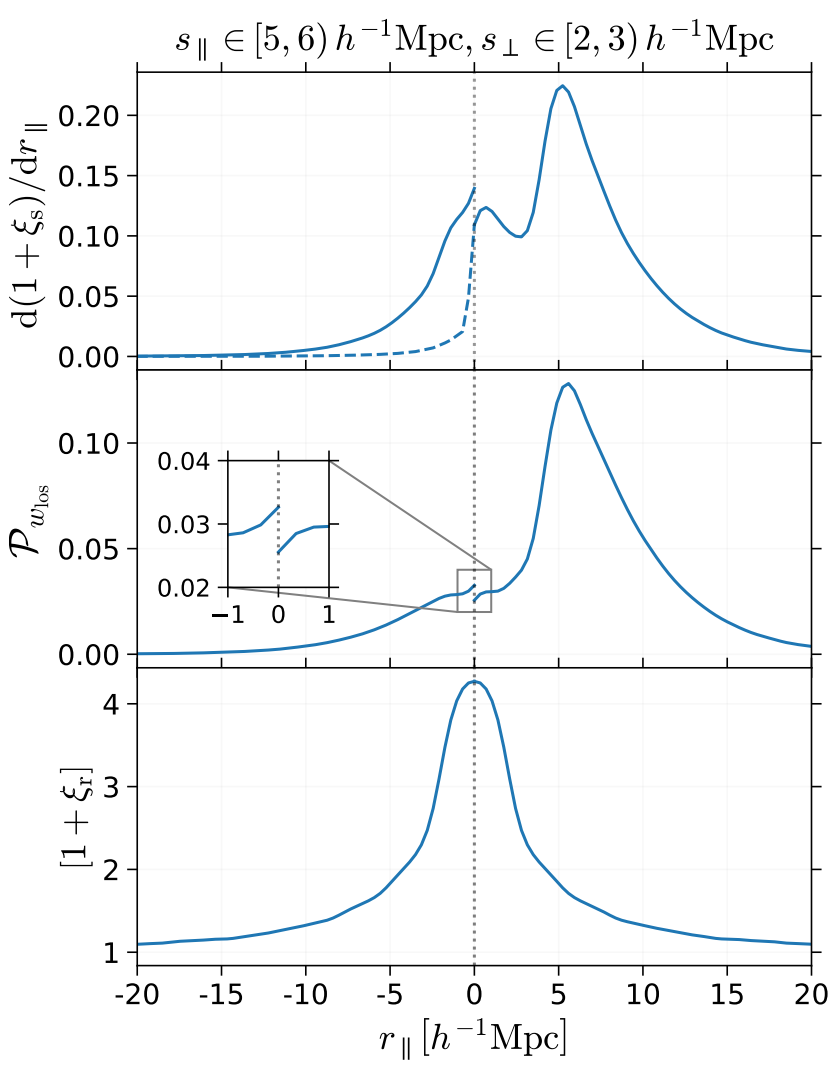

Figure A2. Top: The solid line shows the function $\mathrm{d}\left(1+\xi_{\mathrm{s}}\right) / \mathrm{d} r_{\|}$ i.e. the integrand in equation (22) - for a narrow range of redshiftspace separations indicated above the plot. The dashed line for $r_{\|}<0$ shows the effect of removing the sgn function from the argument of the velocity PDF in equation (22). Note that this severely miscounts the reversed pairs ultimately leading to an underestimate of $\xi_{\mathrm{s}}$. Middle: The contribution to the integrand due to the pairwise-velocity PDF, $\mathcal{P}_{w_{\text {los }}}\left[\left(s_{\|}-r_{\|}\right) \operatorname{sgn}\left(r_{\|}\right) \mid \mathbf{r}\right]$, is plotted as a function of $r_{\|}$. Bottom: The contribution to the integrand due to real-space clustering, $1+\xi(r)$, is shown as a function of $r_{\|}$. 